\title{
An Empirical Modelling for the Baseline Energy Consumption of an NB-IoT Radio Transceiver
}

\author{
Sikandar Zulqarnain Khan, Muhammad Mahtab Alam, Senior Member, IEEE, Yannick Le Moullec, Senior \\ Member, IEEE, Alar Kuusik, Member, IEEE, Sven Pärand, and Christos Verikoukis Senior Member, IEEE
}

\begin{abstract}
NarrowBand Internet of Things (NB-IoT) is an emerging cellular IoT technology that offers attractive features for deploying low-power wide area networks suitable for implementing massive machine type communications. NB-IoT features include e.g. extended coverage and deep penetration for massive connectivity, longer battery-life, appropriate throughput and desired latency at lower bandwidth. Regarding the device energy consumption, NB-IoT is mostly under-estimated for its control and signaling overheads, which calls for a better understanding of the energy consumption profiling of an NB-IoT radio transceiver. With this aim, this work presents a thorough investigation of the energy consumption profiling of the Radio Resource Control (RRC) communication protocol between an NB-IoT radio transceiver and a cellular base-station. Using two different commercial off the shelf NB-IoT boards and two Mobile Network Operators (MNOs) NB-IoT test networks operational at Tallinn University of Technology, Estonia, we propose an empirical baseline energy consumption model. Based on comprehensive analyses of the profile traces from the widely used BG96 NBIoT module operating in various states of the RRC protocol, our results indicate that the proposed model accurately depicts the baseline energy consumption of an NB-IoT radio transceiver while operating at different coverage class levels. The evaluation errors of our proposed model vary between $0.33 \%$ and $15.38 \%$.
\end{abstract}

Index Terms-LPWAN, NB-IoT, Empirical Energy Consumption Model, Power consumption, NB-IoT networks, BG96 chip.

\section{INTRODUCTION}

The third generation partnership project (3GPP) has introduced new cellular technologies to enable a wide range of cellular communications specifically for machine-to-machine and Internet of Things applications. These include LTE-M (Long Term Evolution for Machines) and NB-IoT (NarrowBand-IoT) technologies. On one end, LTE-M includes LC-LTE/MTCe (LTE Cat 0) and eMTC (enhanced Machine Type Communication) technologies (wherein eMTC includes LTE Cat M1 and LTE Cat M2), particularly targeted at applications that require mobility and higher data rates [1]. On the other

This project has received funding partly from the European Union's Horizon 2020 Research and Innovation Program under Grant 668995, 951867, partly from the European Union Regional Development Fund in the framework of the Tallinn University of Technology Development Program 2016-2022, and partly from Estonian Research Council grant PRG667.

Sikandar Zulqarnain Khan, Muhammad Mahtab Alam, Yannick Le Moullec and Alar Kuusik are with Thomas Johann Seebeck Department of Electronics, Tallinn University of Technology, Tallinn, Estonia. Sven Pärand is with Telia Estonia. Christos Verikoukis is with Centre Tecnològic de Telecomunicacions de Catalunya (CTTC), Spain

Copyright (c) 20xx IEEE. Personal use of this material is permitted However, permission to use this material for any other purposes must be obtained from the IEEE by sending a request to pubs-permissions@ieee.org. hand, NB-IoT includes LTE CAT-NB1 and LTE CAT-NB2 technologies, particularly targeted at applications that require lower complexity and lower data rates [2], [3]. Furthermore, both eMTC and NB-IoT are built upon the existing and already deployed 4G LTE infrastructure to support energy-constrained, mostly battery-powered IoT devices [4].

To reduce the power consumption of an end-device, also called a User Equipment (UE), both eMTC and NB-IoT provide extended versions of the existing power saving features of the legacy LTE technology, i.e. eXtended Discontinuous Reception (eDRX) and Power Saving Mode (PSM), to help prolong the UE's battery lifetime [5], [6]. Utilizing these features in the UE requires a Radio Resource Control (RRC) connection setup between the UE and the network; a detailed overview of this RRC protocol is provided in Section II of this paper.

The eDRX feature enables the device to switch off parts of its radio circuitry, thereby operating with limited functionality and thus reduced power consumption [7], making it a useful feature for network-oriented applications where the device can be woken up remotely by the network as needed, e.g., in smartgrid applications. The PSM feature, on the other hand, enables the device to switch off its radio circuity, thereby operating with the lowest possible power consumption [7], making it a useful feature for device-oriented applications where the device is not accessible to the network but is woken up locally as scheduled (time-triggered) by the application e.g., in smartmetering and public-bike-sharing applications, etc.

A typical NB-IoT device include a radio transceiver, a microcontroller, and additional peripherals as its main components; among them, the radio transceiver has significantly higher energy consumption. Thus, understanding the details of the energy consumption of the radio transceiver is an important research topic in order to better estimate the lifetime of NBIoT devices.

\section{A. State of the art}

Several works have evaluated the NB-IoT technology in terms of UE's power consumption analysis and battery lifetime estimations [6-22]; these works can be categorized into analytical, simulations, and experimental measurements based analyses. Most of these works provide analytical models with simulation-based energy estimations [8]-[16]. For example, the work in [8] focus on finding the optimum length of an eDRX cycle to help mitigate the signaling cost in an LTE network with simulations-based analysis. The authors in [10] 
have presented an NB-IoT energy consumption model with uplink and downlink data transmissions as defined by Poisson processes. The authors in [11] and [12] have tried to estimate the NB-IoT device battery life-time by using some simplified energy consumption equations, whereas the authors in [14] have proposed an NB-IoT UE energy consumption analytical model based on Markov chains. Similarly, the work in [16] presents an analytical model for evaluating the latency and maximum number of devices in any network. Overall, most of the analytical models as presented in these works have been validated through network simulators. Such validations have higher uncertainty as the models' estimates and the validations do not use accurate actual measurements.

Several works have also provided experimental power consumption analysis of the NB-IoT technology, such as [17][24]. For example, the work in [17] focuses on the design of an NB-IoT prototype for delay-tolerant applications while operating in different coverage levels of the network. Although this work provides power consumption measurements of the NB-IoT UE as a whole, the individual power consumption details for each state of the operating mode of radio/node are missing. The work in [18] focuses on the latency issues of NB-IoT while making use of a commercial NB-IoT network in Belgium. Although this work provides empirical results for analyzing the network performance in terms of setup times, throughput, and latency, it does not present the power consumption details of the UE. The work in [19] provides empirical results for the current traces of CoTS NB-IoT platform , i.e. Ublox SARA-N211 when operating on Vodafone's network in Barcelona, Spain. While this work provides coarsegrained current traces for the various states of the radio, i.e. Active Waiting (C-DRX mode), Idle waiting (eDRX), and PSM; the underneath fine-grained details for their respective C-DRX cycles, eDRX cycles including Paging Time Windows (PTW) and their underneath I-DRX cycles with sleep periods (SP) and paging occasions (POs) are missing.

The authors in [21] claim to provide the first publicly available empirical power consumption measurements for the NB-IoT devices but their measurement setup is emulated using a Keysight UXM, a standard-compliant NB-IoT BS emulator. That is why it is unclear as to what extent their results would map onto a real network. Similarly, the work in [23] proposes a Dual-RAT LPWAN node combining an NB-IoT and LoRaWAN radio into one node with all the necessary power regulator circuitry. Here too, the power consumption numbers are given for the whole node only, and the individual power graphs for the radio modes and their internal state details are missing.

Considering the above sate-of-the-art and to the best of our knowledge, the following research gaps exist in the literature. First, no detailed baseline power consumption assessment of the NB-IoT radio has yet been provided. Second, an accurate energy consumption model that truly depicts the empirical energy consumption of an NB-IoT radio across its various stages of RRC operation (i.e. attach, active waiting, idle waiting, resume) is missing. Third, recently published works on the NB-IoT UE's power consumption present only a coarsegrain analysis of the NB-IoT node(s), mostly providing the aggregated power consumption of the whole node where the individual power consumption details of the underneath activities remain mostly obscured. In other words, the detailed energy-consumption profiling of the various states of the CoTS NB-IoT radio module(s) and its underneath activities remain unexplored to date. Fourth, most of the existing analyses are based on emulated NB-IoT networks (in particular the base-station (BS)) and not on actual network operating BS. Similarly, the detailed energy consumption profiling of the commercially available (CoTS) NB-IoT devices under real mobile network operators (MNOs) networks are yet to be explored.

\section{B. Contributions}

This work provides a modelling methodology for profiling the baseline energy consumption of an NB-IoT radio transceiver based on detailed empirical measurements. The modelling methodology considers all the states of the RRC protocol standardized by $3 \mathrm{GPP}$ and hence is applicable to general NB-IoT radio chips that are standard compliant.

The main contributions of our paper and its positioning with reference to the state of the art can be summarized as follows:

- Decomposition of the LTE RRC protocol with precise details and experimental demonstrations: while the 3GPP standard documentation ( [6], [25]-[27]) and a number of papers in the literature (among others [19]) present the key concepts of the LTE RRC protocol, to the best of our knowledge, this work is the first one to delve into a fine grain analysis of the LTE RRC protocol while mapping its different stages and modes with equally detailed experimental results in terms of energy consumption, thereby providing details and an understanding of the baseline energy consumption at a level not available so far.

- Empirical and detailed power consumption measurements of CoTS NB-IoT radio transceiver while operating under real networks: in contrast to most existing works (e.g., [19], [21], [23]) that are limited to the aggregated power or energy consumption of the whole NB-IOT UE and/or rely on either simulations or emulated networks, this work analyzes the energy consumption of the radio transceiver in details (i.e. for each state of the RRC protocol) while operating under two MNOs-deployed NB-IoT test networks; this provides not only a more detailed analysis but also more realistic, empiricalbased results as compared to the state of the art.

- Derivation of an accurate energy consumption model for an NB-IoT radio transceiver: existing models are analytical only and/or not detailed enough to reflect all the inner mechanisms at play in the NB-IoT radio. To overcome this gap, and to the best of our knowledge, this paper is the first one to propose a detailed and realistic NB-IoT radio transceiver energy consumption model thanks to the detailed analysis and real-life empirical experiments mentioned above.

- The proposed model is evaluated under real life conditions and we calculate the difference between the energy consumption obtained from the real life deployment versus the energy consumption predicted by using our proposed model. Our results show that the error of the proposed model 
ranges between $0.33 \%$ and $15.38 \%$, with the largest deviations occurring in the attach and resume procedures.

The remainder of this paper is organized as follows. Section II provides an overview of the RRC protocol whereas Section III presents our proposed NB-IoT radio energy consumption model. Section IV presents the empirical measurement results of the NB-IoT radio energy consumption at its various states of operation and Section $\mathrm{V}$ presents the evaluation of the proposed model. Section VI summarizes our conclusions and future works.

\section{Overview of RAdio Resource Control (RRC) PROTOCOL}

The RRC is a communication protocol between an end device/UE and the base-station (also termed evolved Node$\mathrm{B}(\mathrm{eNB}))$ through which network services such as connection establishment, connection maintenance, data exchange, sleep and notification patterns, security and Quality of Service (QoS), etc. take place. The RRC protocol model has only two complementary states , i.e. 1) RRC_Connected and 2) RRC_Idle, as shown in the RRC protocol reference model in Figure 1; the radio alternates between these two states during operation.

As shown in Figure 1, the UE, on power up (or cold start), requests a network connection from the BS which upon acknowledgement is granted network resources and it thus enters into the RRC_Connected state. The connection establishment takes place in the "Attach" procedure and is always initiated by the UE. Once connected, the exchange of (uplink(Tx)/downlink(Rx)) data between the UE and the network takes place in the allocated transmission and reception slots that have been previously allocated to the UE during the "Attach" procedure. After a secure exchange of data, the UE listens to the broadcast information from the eNB for a certain period of time that is termed as "Active waiting" and whose period is set by the network operator. If any data arrives during this period, the RRC connection is resumed for the exchange of data between the UE and the network such that active waiting period restarts at the end of the data exchange. However, if no data arrives during active waiting, the eNB releases the connection and the UE switches to the RRC_Idle state, thereby saving all the context of the network in local memory.

Transiting into RRC_Idle state, the UE may enter either into eDRX or into PSM as per its configuration. The UE can also alternate between these two states, with eDRX first and PSM next, incase if both states are enabled. In the eDRX mode, the UE listens to the broadcast information from the eNB in cyclic patterns known as eDRX cycles; hence this phase is termed as eDRX mode. When the eDRX mode expires, or when it is forced to expire, the UE switches to the PSM mode during which it turns off its radio and is therefore not reachable by the network. This mechanism facilitates the device to enter deeper hardware sleep modes and thus contribute towards maximum power savings of the UE's battery, but at the cost of increased latency.

To summarize, the NB-IoT radio goes through the following states as it operates under the RRC protocol, i.e. (i) Attach
- registration to the network on a cold start or power up, (ii) Data Exchange $(\mathrm{Tx} / \mathrm{Rx})$ - transmission and reception of data to/from the network, (iii) Active Waiting (C-DRX mode) continuous listen to the broadcast information from the eNB for a period as permitted by the network operator and as configured by the UE, (iv) Idle Waiting (eDRX mode) - partly listens to the broadcast information from eNB for a period as permitted by the operator and as configured by the UE, (vi) Power Saving Mode (PSM) - shut-down of the radio activity for a period as requested by the UE and that as acknowledged by the network, and (viii) Tracking Area Update (TAU) resuming the connection with eNB on wake up from PSM. All these radio states are shown in the RRC reference model in Figure 1.

Details of these radio states are discussed in what follows.

\section{A. Attach - RRC_Connected state}

On powering up, the radio scans the air for a suitable network interface through a contention-based Random Access (RA) preamble to which the eNB responds with a Random Access Response (RAR) message. The UE then sends an RRC connection request to which the eNB responds with an RRC connection setup and the UE thus gets connected to the eNB. Afterwards, the UE establishes a connection with the core network and generates an Access Stratum (AS) security context for secure exchange of data. After a successful AS security setup, the eNB reconfigures the RRC connection to finally establish a data radio bearer for the UE to uplink its data packets in the allocated transmission (Tx) slots. Further details on the attach procedure can be found in [26] and [27].

\section{B. Data Exchange $(T x / R x)$ - RRC_Connected state}

When the UE wants to transmit some data to the network, it first establishes an RRC connection with the network through an Attach procedure (on powering up) or TAU procedure (on waking up from PSM) and transits to the RRC Connected state. It then transmits its data packets to the network in its allocated transmission (Tx) slots using some transmission protocols (such as UDP, HTTPS, MQTT, etc). On the other hand, when the network wants to transmit some data to the UE (i.e. the UE will now receive data), there are two possibilities for the network to reach the UE in its RRC_Idle state, depending on whether it is in eDRX or PSM mode. If the $\mathrm{UE}$ is in eDRX mode, it periodically listens to the broadcast messages from the network during the paging occasion (PO) of each I-DRX cycle. In this case, the network sends a paging message to the UE and notifies it of the pending downlink traffic. As the UE interprets the paging message, it initiates a connection resume/reconnect procedure to get connected to the network and thus the exchange of downlink data between the UE and network occurs in the allocated reception (Rx) slots. However, if the UE is in PSM mode, it is not reachable by the network until the expiration of its PSM period (i.e. T3412-T3324). As the PSM expires, the UE initiates the TAU procedure to resume connection with the network, after which the data exchange occur. More details on data exchange can be found in [13] and [28]. 


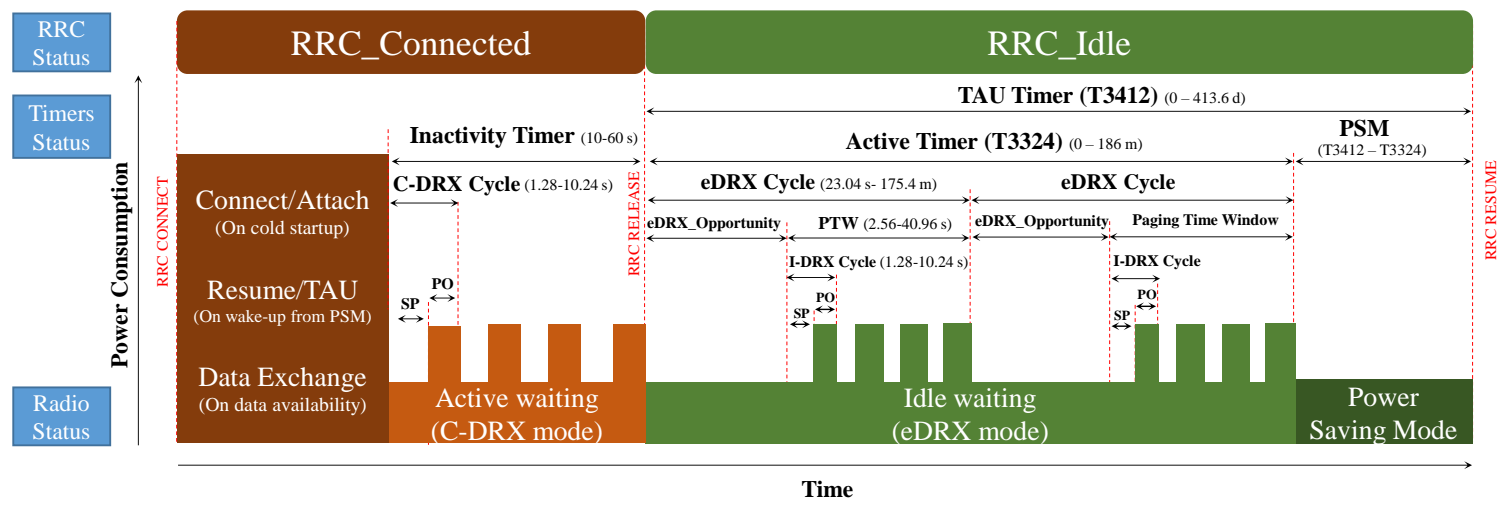

Figure 1: RRC protocol reference model for the NB-IoT radio. It is composed of two complementary states, i.e. RRC_Connected and RRC_Idle and exploits Active waiting, Idle waiting and Power Saving Mode (PSM) after establishing a connection with the network. From top to bottom: (top) RRC connection status, (middle) timers with their minimum and maximum limits, and (bottom) radio status with associated power consumption, as depicted schematically.

\section{Active waiting - RRC_Connected state}

Discontinuous Reception (DRX) is a legacy LTE feature that enables the UE to discontinuously receive the Physical Downlink Control Channel (PDCCH) to maintain network synchronization and determine if there is any pending downlink data. In the LTE RRC protocol, the DRX feature can be enabled both in the RRC_Connected state, i.e. ConnectedDRX (C-DRX), and in the RRC_Idle state, i.e. Idle-DRX (IDRX). In the RRC_Connected state, when there is no data traffic, the UE alternates between a Sleep Period (SP) during which the radio remains quiet and a Paging Occasion (PO), also called Paging Event (PE), during which the radio monitors the PDCCH such that SP and PO alternates in a cyclic pattern that is termed C-DRX cycle (where $\mathrm{C}$ stands for connected state of the radio). These SPs and POs patterns (i.e. C-DRX cycles) repeat for the entire duration of "Active waiting" phase and whose length is controlled by the value of the Inactivity Timer. The value of the Inactivity Timer is operator specific (10-60 s in most commercial networks) and the UE cannot override its value as set by the operator. Furthermore, the Inactivity Timer starts running automatically; either at the end of data exchange between the UE and the network or when no data is available after the Attach procedure where upon its expiration the network releases the connection and the device switches to RRC_Idle state [25]. If some data arrives while the UE is still active waiting (i.e., Inactivity Timer is running), the connection is resumed for the exchange of data between the UE and the network; the Inactivity Timer restarts at the end of this data exchange and the UE enters into its active waiting phase again.

\section{Idle waiting (eDRX mode) - RRC_Idle state}

In the RRC_Idle state, new resources cannot be requested from the network. However, the UE is still reachable by the network where it periodically monitors the Physical Downlink Control Channel (PDCCH) in cyclic patterns. The NPDCCH monitoring takes place during the on-phase of an I-DRX cycle (where I stands for Idle state of the radio), i.e. PO or PE, whereas during the next off-phase of the I-DRX cycle, i.e. $\mathrm{SP}$, the radio does not perform any activity. These I-DRX cycles repeat for the entire duration of a paging time window (PTW). A PTW itself forms the active phase of an eDRX Cycle such that each PTW is followed by an inactive phase that is termed as an eDRX_Opportunity and during which the radio remains inoperative for until the beginning of the next PTW. These cyclic patterns of eDRX_Opportunity and PTW, i.e eDRX Cycles occur repeatedly during the entire span of the Idle waiting state of the radio. Since idle waiting involves repeated eDRX cycles, this phase is also termed eDRX mode. All these nested cycles of activity and inactivity periods occurring during the eDRX mode are shown in the RRC protocol reference model in Figure 1.

The eDRX mode is controlled by a set of timers where the active timer (i.e. T3324) primarily controls the time lapse of the entire duration of the eDRX mode and can have an extended range from 0 to $186 \mathrm{~min}$ for NB-IoT, with a maximum period of 175.4 min for its eDRX cycle and a maximum period of $40.96 \mathrm{~s}$ for its PTW. The maximum IDRX cycle can be of $10.24 \mathrm{~s}$ for NB-IoT. The minimum and maximum limits of these cycles for NB-IoT technology are also indicated in the RRC protocol reference model in Figure 1. Further details on their minimum and maximum ranges can be found in [6], [7]. It is worth mentioning here that the UE can configure the length of its eDRX mode, the length of its eDRX cycle, and the duration of its PTW, only if permitted by the network.

\section{E. PSM - RRC_Idle state}

On expiration of the active (T3324) timer, the UE exits idle waiting (eDRX mode) and enters into a Power Saving Mode (PSM). While in PSM, the UE turns its radio off for as long as the TAU timer is running and its energy consumption approaches to almost that of its power-off state. It is worth noting that though the radio or UE is not reachable by the 
network, it is still registered with the network, so that when the UE wakes-up from PSM, it does not have to go through the registration process all over again; this adds to a significant amount of energy savings in reducing the signaling overhead. Further details on the resume procedure can be found in [26], [27] and [15], [28].

As the TAU (T3412) timer expires, the PSM is exited and the UE wakes up to perform the "Tracking Area Update (TAU)" procedure when the already registered UE reconnects with the network to check for any pending uplink/downlink data. Once this data exchange has occurred, the active waiting period restarts; when it ends, the UE enters into the RRC_Idle state and the cycle repeats. It should be noted here that the PSM mechanism implies a low power consumption at the cost of higher latency. Because the network has to wait until the UE is up again from its PSM and reachable by the network. As NB-IoT is designed for latency-tolerant applications, the UE may (deep) sleep for an extended range of up to 413 days and still be registered with the network. More details on the PSM state can be found in [6], [19].

\section{F. Tracking Area Update (TAU) - RRC_Connected state}

On expiration of the TAU (T3412) timer, the device wakes-up from its PSM and reconnects to to the network to indicate its availability in the tracking area update (TAU) procedure. During the TAU procedure, the UE listens to any scheduled DL data that, if exists, is downloaded in the allocated reception $(\mathrm{Rx})$ slots. Similarly, if the UE has any UL data, it is transferred to the network in the allocated transmission (Tx) slots. If no data exists for exchange, the Inactivity timer starts so that the device enters into active waiting phase. As it finishes, the device enters into idle waiting and the cycle continues. Further details on the TAU procedure can be found in [6], [26], [27].

This section has presented an in-depth analysis of the NBIoT RRC protocol phases; thanks to this knowledge, we can now proceed with building an empirical NB-IoT UE energy consumption model, which we describe in the next sections.

\section{Proposed Model for Profiling the Baseline ENERGY CONSUMPTION OF NB-IOT RADIO TRANSCEIVER}

In addition to the detailed analysis of the RRC protocol presented in the previous section, an empirical model that provides a detailed baseline energy consumption of the RRC protocol is presented in this section.

Since the RRC protocol has only two states, i.e. 1) RRC_Connected and 2) RRC_Idle, the total energy consumed by an $\mathrm{RRC}$ radio can be given as:

$$
E_{T O T A L}=E_{R R C \_C O N N E C T E D}+E_{R R C_{-} I D L E}
$$

In the RRC_Connected state, the radio goes through the four following states, i.e. Attach, Data Exchange (Tx/Rx), Active waiting (C-DRX) and TAU. The Attach procedure occurs only after a cold start whereas the TAU procedure occurs each time the radio wakes up from PSM. Thus, the total energy consumed during the RRC_Connected state can be written as:

$$
\begin{aligned}
E_{R R C_{-} C O N N E C T E D}= & E_{A T T A C H}+E_{T x / R x} \\
& +E_{C-D R X}+E_{T A U}
\end{aligned}
$$

As the Inactivity Timer finishes, the RRC connection is released and the radio goes into the $\mathrm{RRC}$ _Idle state where the radio first enters into Idle waiting state or eDRX mode, followed by PSM. Thus, the total energy consumed during the RRC_Idle state can be written as:

$$
E_{R R C_{-} I D L E}=E_{e D R X}+E_{P S M}
$$

Since Energy $=$ Power $\times$ Time, the average energy consumption during the RRC_Connected state can be written as:

$$
\begin{array}{r}
E_{R R C \_C O N N E C T E D}= \\
\left\{P_{A T T A C H(\text { avg })} \times T_{A T T A C H}\right\}+\left\{\left(P_{T x(a v g)} \times T_{T x}\right)\right. \\
\left.+\left(P_{R x(\text { avg })} \times T_{R x}\right)\right\}+\left\{P_{C-D R X(a v g)}\right. \\
\left.\times T_{\text {InactivityTimer }}\right\}+\left\{P_{T A U(\text { avg })} \times T_{T A U}\right\}
\end{array}
$$

Since the ActiveW aiting (C-DRX mode) period is a series of repeated C-DRX cycles, the above equation can be rewritten as:

$$
\begin{array}{r}
E_{R R C \_C O N N E C T E D}=\left\{P_{A T T A C H(\text { avg })} \times T_{A T T A C H}\right\}+ \\
\left\{\left(P_{T x(\text { avg })} \times T_{T x}\right)+\left(P_{R x(\text { avg })} \times T_{R x}\right)\right\}+\left\{P_{C-D R X(\text { avg })}\right. \\
\left.\times\left(T_{C D R X_{-} \text {Cycle }} \times N_{C D R X_{-} \text {Cycles }}\right)\right\}+\left\{P_{T A U(\text { avg })} \times T_{T A U}\right\}
\end{array}
$$

where $T_{C D R X \_C y c l e}$ is the time period of each C-DRX cycle, and $N_{C D R X_{-} C y c l e}$ is the total number of C-DRX cycles that occur during the ActiveWaiting period.

Similarly, the average energy consumption of the radio during the RRC_Idle state is:

$$
E_{R R C_{-} I D L E}=E_{e D R X}+E_{P S M}
$$

and can be re-written as:

$$
\begin{array}{r}
E_{R R C_{-} I D L E}= \\
\left\{P_{e D R X(a v g)} \times T_{e D R X}\right\}+\left\{\left(P_{P S M(a v g)} \times T_{P S M}\right\}\right.
\end{array}
$$

The duration of the entire Idle state of the radio, and its eDRX and PSM durations, can be set by the values of 3GPP specified timers, such that:

$$
\begin{gathered}
T_{R R C \_I D L E}=T_{3412} \\
T_{e D R X}=T_{3324} \\
T_{P S M}=T_{3412}-T_{3324}
\end{gathered}
$$

Thus, the average energy consumption of the radio during the RRC_Idle state can be re-written as:

$$
\begin{array}{r}
E_{R R C_{-} I D L E}=\left\{P_{\text {eDRX }(\text { avg })} \times T_{3324}\right\} \\
\quad+\left\{P_{P S M(\text { avg })} \times\left(T_{3412}-T_{3324}\right)\right\}
\end{array}
$$

Since, the $e D R X$ mode is composed of repeated $e D R X$ cycles, thus: 


$$
\begin{aligned}
E_{R R C_{-} I D L E}=\left\{P_{e D R X(a v g)}\right. & \left.\times\left(T_{e D R X_{-} C y c l e} \times N_{e D R X_{-} C y c l e s}\right)\right\} \\
& +\left\{\left(P_{P S M(a v g)} \times\left(T_{3412}-T_{3324}\right)\right\}\right.
\end{aligned}
$$

where $T_{e D R X_{-} C y c l e}$ is the time period of each eDRX cycle and $N_{e D R X_{-} \text {Cycles }}$ is the total number of eDRX cycles that occur during the IdleWaiting period.

Since each eDRXcycle is composed of a PTW (active phase of an $e D R X$ cycle) and $e D R X \_o p p o r t u n i t y$ (inactive phase of an $e D R X$ cycle), the above equation can be expanded to:

$$
\begin{aligned}
E_{R R C_{-} I D L E}=\left\{P_{e D R X(\text { avg })} \times\right. & \left(T_{e D R X_{-} P T W}+\right. \\
\left.\left.T_{e D R X_{-} O P P}\right) \times N_{e D R X_{-} C y c l e s}\right\} & +\left\{\left(P_{P S M(\text { avg })} \times\right.\right. \\
& \left.\left(T_{3412}-T_{3324}\right)\right\}
\end{aligned}
$$

Moreover, since the power consumption of $P T W$ and $e D R X \_o p p o r t u n i t y$ during each $e D R X$ cycle is different, the above equation can be written as:

$$
\begin{array}{r}
E_{R R C_{-} I D L E}=\left\{\left(P_{e D R X_{-} P T W(a v g)} \times T_{e D R X_{-} P T W}\right)\right. \\
\left.+\left(P_{e D R X_{-} O P P(a v g)} \times T_{e D R X_{-} O P P}\right) \times N_{e D R X_{-} C y c l e s}\right\} \\
+\left\{\left(P_{P S M(a v g)} \times\left(T_{3412}-T_{3324}\right)\right\}\right.
\end{array}
$$

As $P T W$ is a repeated sequence of $I-D R X$ cycles, the above becomes:

$$
\begin{array}{r}
E_{R R C_{-} I D L E}=\left\{\left(P_{\text {eDRX_PTW }(\text { avg })} \times\left(T_{I-D R X_{-} C y c l e}\right.\right.\right. \\
\left.\times N_{I-D R X_{-} C y c l e s}\right) \\
+\left(P_{\text {eDRX_OPP }(\text { avg })} \times T_{\text {eDRX_OPP }}\right) \\
\left.\times N_{\text {eDRX_Cycles }}\right\} \\
+\left\{\left(P_{P S M(\text { avg })} \times\left(T_{3412}-T_{3324}\right)\right\}\right.
\end{array}
$$

where $T_{I-D R X_{-} C y c l e}$ is the time period of each $I-D R X$ cycle and $N_{I-D R X \_C y c l e s}$ is the total number of $I-D R X$ cycles occurring during the PTW of each eDRXcycle.

Next, since each $I-D R X$ cycle has an on phase (i.e. PO) during which the NPDSCCH signal is monitored and an off phase with no activity, the above equation can be expanded to:

$$
\begin{array}{r}
E_{R R C \_I D L E}=\left\{\left(P_{I-D R X_{-} o n(a v g)} \times T_{I-D R X_{-} o n}\right)+\right. \\
\left.\left(P_{I-D R X_{\_} o f f(a v g)} \times T_{I-D R X_{-} o f f}\right) \times N_{I-D R X_{-} C y c l e s}\right) \\
\left.+\left(P_{\text {eDRX_OPP }(a v g)} \times T_{e D R X_{-} O P P}\right) \times N_{e D R X_{-} C y c l e s}\right\} \\
+\left\{\left(P_{P S M(a v g)} \times\left(T_{3412}-T_{3324}\right)\right\}\right.
\end{array}
$$

Finally, given that

$$
E_{T O T A L}=E_{R R C_{-} C O N N E C T E D}+E_{R R C \_R E L E A S E D}
$$

we obtain:

$$
\begin{array}{r}
E_{T O T A L}=\left\{\left\{P_{A T T A C H(a v g)} \times T_{A T T A C H}\right\}+\left\{\left(P_{T x(a v g)} \times T_{T x}\right)\right.\right. \\
\left.+\left(P_{R x(a v g)} \times T_{R x}\right)\right\}+\left\{P_{C-D R X(a v g)} \times\left(T_{C D R X_{-} C y c l e}\right.\right. \\
\left.\left.\left.\times N_{C D R X_{-} C y c l e s}\right)\right\}+\left\{P_{T A U(\text { avg })} \times T_{T A U}\right\}\right\}+ \\
\left\{\left\{\left\{\left(P_{I-D R X_{\text {on }(\text { avg })}} \times T_{I-D R X_{o n}}\right)+\left(P_{I-D R X_{o f f(a v g)}} \times T_{I-D R X_{o f f}}\right)\right.\right.\right. \\
\left.\times N_{I-D R X_{-} C y c l e s}\right\}+\left\{\left(P_{e D R X_{-} O P P(\text { avg })} \times T_{\left.e D R X_{-} O P P\right)}\right\}\right. \\
\left.\left.\times N_{e D R X_{-} C y c l e s}\right\}+\left\{P_{P S M(a v g)} \times\left(T_{3412}-T_{3324}\right)\right\}\right\}
\end{array}
$$

For simplicity, the above equation can be rearranged in terms of the 3GPP specified timers such that each row in the following equation represents the energy consumption of each separate state of the radio, i.e. Attach, Data Exchange (Transmit (Tx) and Receive (Rx)), Active waiting (C-DRX mode), Idle waiting (eDRX mode), deep sleep mode (PSM) and Resume (TAU), i.e.

$$
\begin{gathered}
E_{T O T A L}= \\
\left(\begin{array}{c}
\left\{P_{A T T A C H(\text { avg })} \times T_{A T T A C H}\right\}+ \\
\left\{\left(P_{T X(a v g)} \times T_{T x}\right)+\left(P_{R x(a v g)} \times T_{R x}\right)\right\}+ \\
\left\{P_{C-D R X(\text { avg })} \times\left(T_{\text {InactivityTimer }}\right)\right\}+ \\
\left\{P_{T A U(\text { avg })} \times T_{T A U}\right\}
\end{array}\right) \\
+\left(\begin{array}{c}
\left\{\left(P_{\text {eDRX }(\text { avg })} \times T_{3324)}\right\}+\right. \\
\left\{P_{P S M(\text { avg })} \times\left(T_{3412}-T_{3324}\right)\right\}
\end{array}\right)
\end{gathered}
$$

This section has presented the proposed NB-IoT UE energy consumption model. The next sections detail the corresponding results and corresponding evaluations.

\section{EMPIRICAL MEASUREMENTS}

As explained in Section I-A, works on experimental energy consumption profiling of NB-IoT radio transceivers are limited. To overcome the limitations of the state of the art, a comprehensive model for profiling the empirical energy consumption of an NB-IoT radio transceiver using RRC protocol has been proposed in the previous section. The proposed model relies on empirical measurements; this section presents our experimental setup and measurement results obtained with two widely used CoTS NB-IoT radio boards (both equipped with BG96 module) with network configurations from two MNOs (referred to as Operator 1 and Operator 2) operating NB-IoT test networks at Tallinn University of Technology.

\section{A. Experimental Setup}

The two CoTS NB-IoT radio modules, i.e. Avnet Silica NB-IoT sensor shield [29] and Quectel UMTS \& LTE EVB Kit [30] are based on 3GPP Rel-13 compliant Quectel BG96 LPWAN module [31]. They are used for conducting the current and power consumption measurements while in actual operation under the two publicly available test networks provided by Operator 1 and Operator 2 .

A Keysight Technologies N6705C DC Power Analyzer (PA) [32] is used for collecting the current and power traces during 


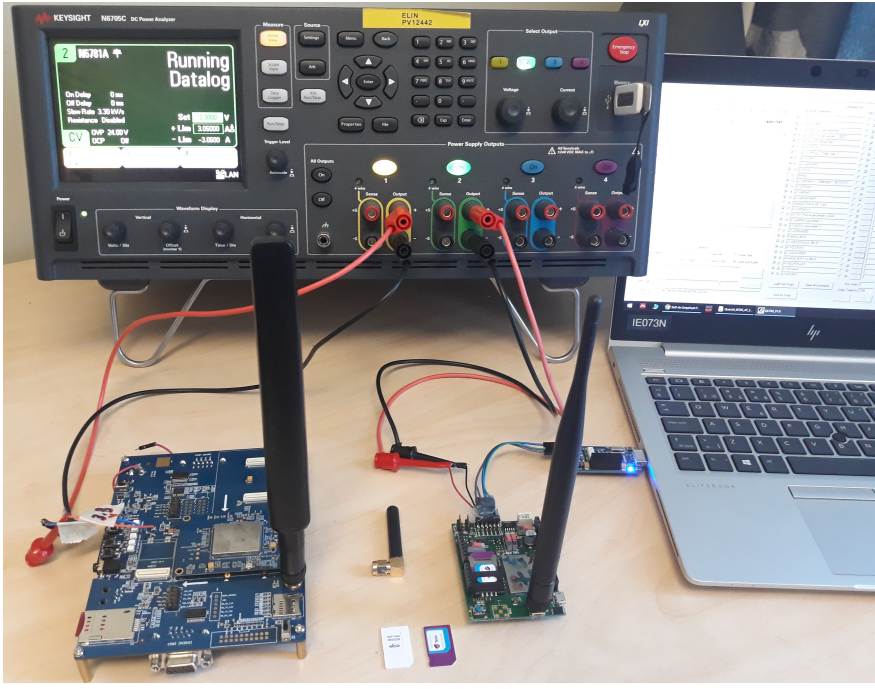

Figure 2: Testbed Setup with Quectel BG96 and Avnet Silica BG96 NB-IoT radio modules, Keysight N6705C DC Power Analyzer, and SIM cards from Operator 1 and Operator 2.

Table I: Details of the publicly available NB-IoT networks that have been used during our measurement campaigns on test location

\begin{tabular}{|c|c|c|}
\hline Details & Operator 1 & Operator 2 \\
\hline Operator numeric code & 24801 & 24802 \\
\hline Selected Access Technology & CAT-NB1 & CAT-NB1 \\
\hline Selected Band & LTE BAND 20 & LTE BAND 20 \\
\hline Selected Channel ID & 6254 & 6152 \\
\hline CE level (at test locations) & 0,1 & \\
\hline $\operatorname{SNR}\{0$ (bad) to 31 (good) $\}(\mathrm{dB})$ & 28,6 & (avg) \\
\hline $\operatorname{SINR}\{0(\mathrm{bad})$ to 250 (good) $\}(\mathrm{dB})$ & 185,178 (avg) & 153,142 (avg) \\
\hline $\operatorname{RSSI}\{-110$ (bad) to -60 (good) $\}(\mathrm{dBm})$ & $-67,-101$ (avg) & $-72,-110$ (avg) \\
\hline $\operatorname{RSRP}\{-140$ (bad) to -44 (good) $\}(\mathrm{dBm})$ & $-67,-111($ avg) & $-74,-117$ (avg) \\
\hline $\operatorname{RSRQ}\{-19.5(\mathrm{bad})$ to -3 (good) $\}(\mathrm{dB})$ & $-3,-9 \quad$ (avg) & $-3,-10 \quad$ (avg) \\
\hline
\end{tabular}

Table II: Operator specific and UE configurable parameters

\begin{tabular}{lll}
\hline Network Params & Symbol & Value \\
\hline Attach & T_ATTACH & Network_conditions \\
Inactivity Timer & InactivityTimer & Operator_defined \\
C-DRX Cycle & CDRX_Cycle & Operator_defined \\
RRC_Idle & RRC_Idle & UE defined = T3412 Timer value \\
Active Timer & T3324 Timer & UE defined = T3324 Timer value \\
eDRX Cycle & eDRX_Cycle & Network defined; UE configurable \\
PagingTimeWindow & PTW & Network defined; UE configurable \\
eDRX_Opportuity & eDRX_Opp & (eDRX_Cycle - PTW) \\
I-DRX Cycle & I-DRX_Cyc & Operator_defined \\
PowerSavingMode & $P S M$ & UE defined = (T3412-T3324) value \\
\hline
\end{tabular}

these measurement campaigns. Our test-bed setup, composed of an Avnet shield as our device under test (DUT) DUT1 and Quectel EVB Kit as our DUT2, along with the Keysight's PA, is shown in Figure 2. A constant voltage of $3.3 \mathrm{~V}$ is supplied to DUT1 and $3.8 \mathrm{~V}$ to DUT2 by the PA. AT commands are sent from the QCOM software running on the PC through the USB-PMOD interface for DUT1 and through the USB interface, configured accordingly, for DUT2. SIM cards for both the networks under test are also visible in our setup, as shown in Figure 2.

From a practical perspective, it should be mentioned that though the BG96 module of both DUTs were flashed with the latest firmware (FW) version, setting up the (T3324/T3412) timers to our desired values was a cumbersome procedure. Upon contacting Quectel, it turned out that even the latest FW (i.e. BG96MAR02A07M1G) has updates in the form of sub-versions; installing the latest sub-version (i.e. BG96MAR02A07M1G_01.016.01.016) solved most of the Timers' related issues. Similarly, the built-in USB-USB interface on DUT1 that is provided to receive power and AT commands from a PC disrupted the power measurements from the PA. To avoid these disruptions, we used an FTDI chip based serial communication interface to utilize its built-in USB-UART PMOD interface [33] and bypassed its USB-USB port. We also disabled all the functional LEDs [34] of DUT1 to get accurate power consumption measurements from the PA. As for testing DUT2, we also modified it as per the documents provided to us by the Quectel Team. Finally, the details of the two MNOs NB-IoT test networks that have been considered for carrying out this research are summarized in Table I.

During our measurement campaign, the NB-IoT devices were placed at different locations inside Thomas Johann Seebeck Department of Electronics building and students' dormitory building that are located on TalTech campus. For triggering the different coverage levels, the devices were deployed on the second basement (B2) floor of Thomas Johann Seebeck Department of Electronics building where the received signal strength is lower than on the upper floors. On each test location, the current value of the $\mathrm{CE}$ level was queried using the adequate AT commands and when the required CE levels were achieved, i.e. CE Level 0 and CE Level 1, the measurements were made accordingly.

Small differences between the two operators in terms of their SNR, SINR, RSSI, RSRP, and RSRQ for the same CE levels could be observed from their respective values as given in Table I. However, to smooth out the minor variations of the individual results for SNR, SINR, RSSI, RSRP, and RSRQ; their experiments were repeated in the order of 100 times on each test location and the obtained results were averaged into their final values, under their respective $\mathrm{CE}$ levels, as summarized in Table I. Table II summarizes the network parameters that are operator specific and/or UE configurable with a short description of their control and possible values.

\section{B. Measurements Approach}

The Data Logger function of the Keysight PA records the output (voltage, current, and power) data logs of the arbitrary waveform at a sampling rate of $50 \mathrm{kHz}$. The display of the PA can be configured to examine these waveforms with a precision of up to $20 \mu \mathrm{s}$. For example, in Figure 3, the waveform of the power consumption of BG96 radio under real network is recorded as a data log file from the Keysight PA. This data $\log$ file is displayed in the "Maker View" of the data logger screen where the power trace P1 (Label 3 in Figure 3 ) is displayed with $100 \mathrm{~mW} / \mathrm{Div}$ (Label 1 in Figure 3) on vertical/power scale and $20.0 \mathrm{~s} / \mathrm{d}$ (Label 4 in Figure 3 ) on the horizontal/time scale of the PA screen. The voltage (V1) and current (I1) (under Label 1 in Figure 3) are not selected for readability. The markers $\mathrm{m} 1$ and $\mathrm{m} 2$ (Label 2 in Figure 3) are 
set to positions where they intersect the P1 trace of the BG96 radio at the beginning and end of its $\mathrm{C}$-DRX mode (Active waiting); thus, the information available under Labels 5 to 10 presents the data available between $\mathrm{m} 1$ and $\mathrm{m} 2$ markers and can be read as summarized in Table III.

All the measurement results presented in rest of this paper are recorded as data log files and displayed in the Marker view of the PA, similar to the one as shown in Figure 3. This approach is used to produce actual power traces of the BG96 radio under real network with on-field measurements from the PA. For all the power measurements and energy calculations for rest of the waveforms/traces in this paper, Label 9 provides an average power consumption and average timings between the $\mathrm{m} 1$ and $\mathrm{m} 2$ markers. These markers are set to various positions on the respective power traces of the BG96 radio transceiver, so as to obtain the adequate power consumption and timings details for the various states of its RRC operation.

\section{Empirical Results}

A number of experiments were conducted using two CoTS NB-IoT radio modules operating under two MNOs operating NB-IoT test networks in Tallinn, Estonia. To verify and evaluate the correctness of our proposed model, various timings for the different states of the NB-IoT radio modules were tried and tested for different power saving schemes. The generated results were tested for various versions of the FWs of these radio modules to verify their impact on the performance of the NB-IoT radio as they are continuously updated and to see to what extent they are compliant with the 3GPP defined NB-IoT standards. Our obtained results from these tests are explained in the subsections that follow.

1) Testing active waiting (C-DRX) mode of the Avnet BG96 radio under Operatorl network: To evaluate the detailed energy consumption of the C-DRX mode of BG96 radio, we set the network parameters as C-DRX $=1$, eDRX $=0$ and PSM $=0$ and obtained our empirical results for Operator 1 network, as shown in Figure 4. It could be observed that Operator1 had no limitations on the duration of its C-DRX mode as the radio remains in its active waiting state for as long as it was powered on. This is shown in Figure $4 \mathrm{~b}$ where the average power consumption for the entire C-DRX mode is measured to be $0.082 \mathrm{~W}$. Figure 4c details each C-DRX cycle of 2.56 $\mathrm{s}$ with an average power consumption of $0.082 \mathrm{~W}$. Figure $4 \mathrm{a}$ details the attach procedure of the BG96 radio with Operator1 network with an average power consumption of $0.18 \mathrm{~W}$ over $18.6 \mathrm{~s}$.

During the second phase of the same experiment, the C-DRX mode of the BG96 radio was limited to a duration of $1 \mathrm{~m}$, after which the radio was forced to switch to its PSM state, as shown in Figure 5. The respective average power consumption for the C-DRX mode and C-DRX cycle, as shown in Figure 5a and Figure 5c, were found to be the same as previously. However, the average power consumption of the PSM of the BG96 radio was found to be $0.19 \mathrm{~mW}$, as shown in Figure 5b.
2) Testing idle waiting (eDRX) mode of Avnet $B G 96$ radio under Operatorl network: To evaluate the fine-grain energy consumption of the eDRX mode of the BG96 radio with the underneath details of its eDRX cycle(s) that includes an eDRX_Opportunity and a PTW, and the underneath IDRX cycles of each PTW, we carried out a second series of experiments where we set the network parameters as C-DRX = 0 , $\operatorname{eDRX}=1$, and PSM $=1$ with T3324 timer $=4 \mathrm{~m}$; such that the eDRX mode runs for 4 min and then switches to its PSM state. Our results from these experiments are summarized in Figure 6 . The average power consumption for the entire eDRX mode was found to be $0.071 \mathrm{~W}$, as shown in Figure 6a, 0.070 $\mathrm{W}$ for each eDRX cycle of $41.40 \mathrm{~s}$, as shown in Figure $6 \mathrm{~b}$, and $0.078 \mathrm{~W}$ for the PTW of $19.80 \mathrm{~s}$ each, as shown in Figure $6 \mathrm{c}$. The I-DRX cycle was found to be $2.56 \mathrm{~s}$ with an average power consumption of $0.081 \mathrm{~W}$, as shown in Figure $6 \mathrm{~d}$.

3) Testing power cycle (a repeated sequence of $C$-DRX, $e D R X$, and PSM) of the Avnet BG96 radio under Operatorl network: In these set of experiments, we evaluated the finegrain energy consumption of the BG96 radio in a power cycle consisting of C-DRX mode, eDRX-Mode, and PSM with the T3324 timer set to 4 min and T3412 timer set to $1 \mathrm{~h}$; the results are shown in Figure 7. All the obtained results were found to be the same as in the previous Experiment 1 and Experiment 2. Furthermore, it was observed that the radio automatically woke up from its PSM to re-attach with the network and repeat its power cycle with its previous settings. The power traces for the C-DRX, eDRX and PSM states during these experiments are shown in Figures $7 \mathrm{a}, 7 \mathrm{~b}$, and 7c, respectively.

Furthermore, we transmitted 10 bytes of data from the BG96 radio on Operator1 network using UDP protocol at different coverage levels (CEL), as shown in Figure 8a, 8b. It was observed that the radio consumed $0.000372 \mathrm{Wh}$ to transmit data at CEL $=0$ whereas it consumed $0.000816 \mathrm{Wh}$ to transmit the same data at $\mathrm{CEL}=1$, i.e. an increase of $124.09 \%$.

4) Testing power cycle of the Avnet BG96 radio under Operator2 network: All the above experiments were repeated with the Avnet BG96 shield under similar conditions but this time with Operator 2's network. The obtained results from these tests are summarized in Figure 9. During these tests, it was observed that Operator 2's network had more restrictions on their network parameters as compared to Operator 1, i.e. the UE/radio had little provisions to configure the network parameters. For example, the C-DRX mode was fixed to $34 \mathrm{~s}$ (during all our tests) whereas the eDRX mode and PSM could be configured by the UE as desired. However, the eDRX cycle and its underneath PTW in the C-DRX mode could not be configured (contrary to the case with Operator 1). It was also noted that the radio took $12.6 \mathrm{~s}$ on average to get connected to Operator 2's network, as compared to an average of $18 \mathrm{~s}$ on Operator 1's network.

Furthermore, we transmitted 10 bytes of data from the BG96 radio on Operator 2's network using the UDP protocol at different coverage levels, as shown in Figures 10a and $10 \mathrm{~b}$. It was observed that the radio consumed $0.00011 \mathrm{Wh}$ at 


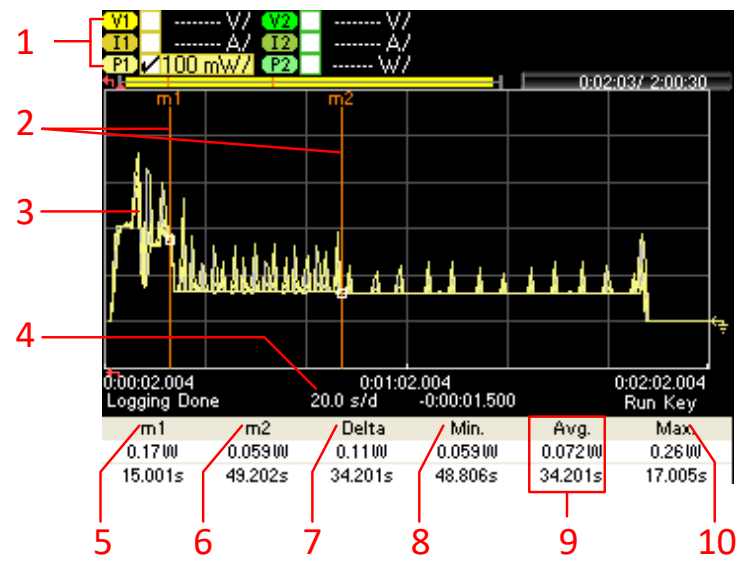

Figure 3: Measurement Setup with Keysight N6705C DC Power Analyzer: Example of an NB-IoT waveform and measurement information available in the Marker View.

Table III: Reading Data from the Marker View of the Power Analyzer

\begin{tabular}{ll}
\hline Symbol/Field & Description \\
\hline 1 Trace Controls & $\begin{array}{l}\text { Identifies the voltage/div. or current/div. settings. Tick }(\sqrt{ }) \text { indicates the trace is on. Dots }(\cdots) \text { indicate the trace } \\
\text { is off. In current setup, we only select the power trace. }\end{array}$ \\
Shows where the measurement markers intersect the selected waveform. Data values at the bottom of the display \\
(i.e. labelled 5-10) are referenced to the intersect locations of the markers. Calculations are based on the data \\
points in between the intersect locations. \\
Voltage, Current, Power trace as selected in Label 1.
\end{tabular}

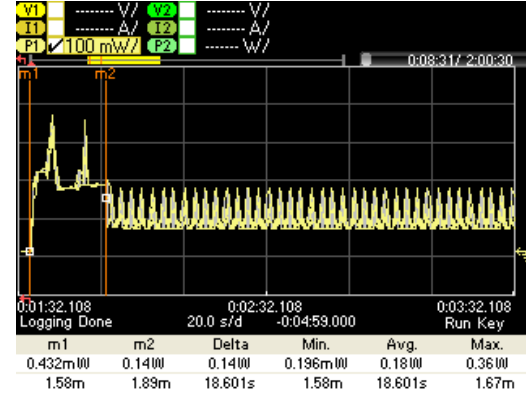

(a) Connect/Attach Mode

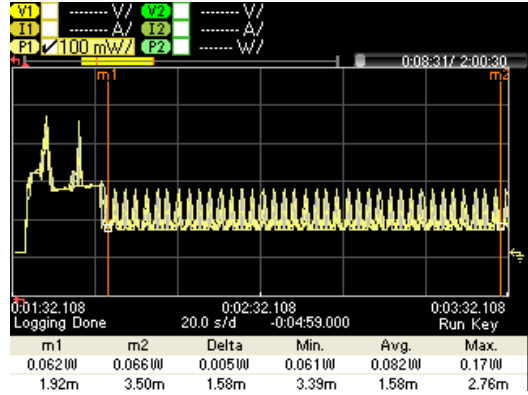

(b) Active waiting (C-DRX Mode)

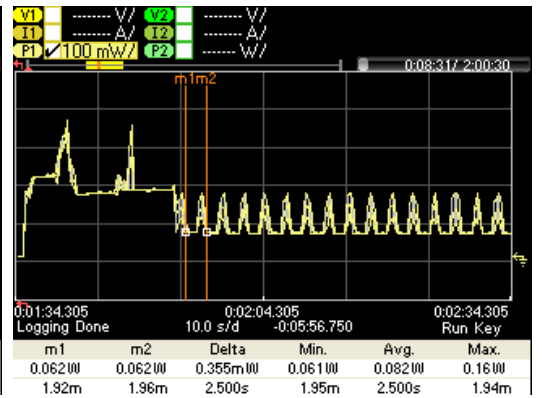

(c) C-DRX Cycle

Figure 4: Continuous CDRX Mode with BG96/Avnet shield under Operator 1: (a) Power trace of UE's Attach procedure with an average power consumption of $0.18 \mathrm{~W}$; (b) Power trace of C-DRX mode with an average power consumption of $0.082 \mathrm{~W}$; (c) Power trace of UE's C-DRX cycle with an average power consumption of $0.082 \mathrm{~W}$.

$\mathrm{CEL}=0$ whereas it consumed $0.00016 \mathrm{Wh}$ to transmit the same data at $\mathrm{CEL}=1$, i.e. an increase of $45.45 \%$. Similarly, a comparison between the effects of overheads involved in the two data transmission protocols (i.e,. UDP and HTTPs) on the energy consumption of the radio was also made, where a desired data of 10 bytes (that was required to be sent from the radio) was transmitted from the BG96 radio on Operator 2's network at different coverage levels, with additional 61 bytes of data that was a requirement of the HTTPs protocol for its server setup. The obtained power 


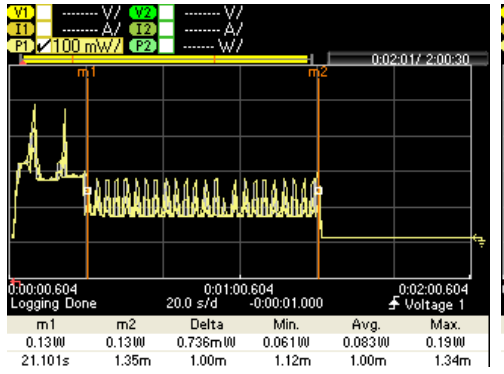

(a) C-DRX Mode (controlled)

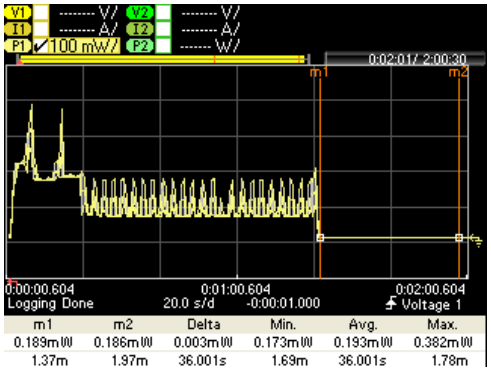

(b) PSM

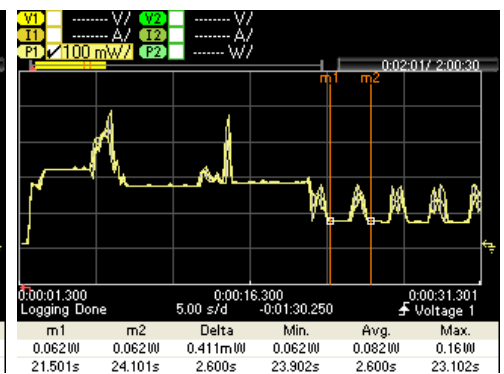

(c) C-DRX Cycle

Figure 5: Controlled C-DRX Mode with BG96/Avnet shield under Operator1: (a) Power trace of UE's C-DRX mode with an average power consumption of $0.083 \mathrm{~W}$; (c) Power trace of UE's PSM with an average power consumption of $0.19 \mathrm{~mW}$; (d) Power trace of UE's C-DRX cycle with an average power consumption of $0.082 \mathrm{~W}$.

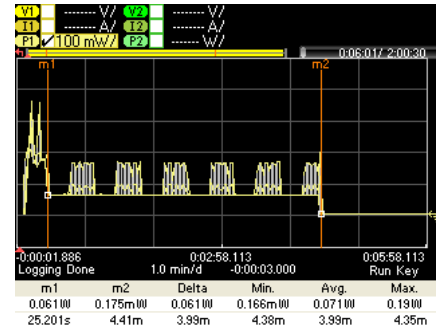

(a) eDRX Mode

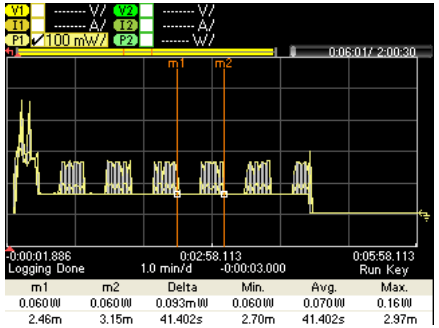

(b) eDRX Cycle

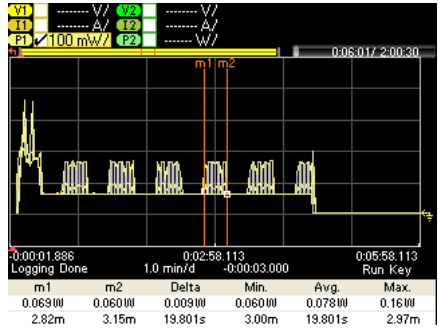

(c) PTW

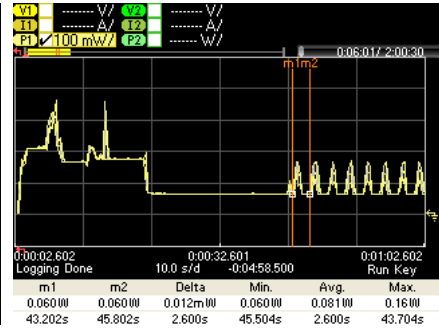

(d) I-DRX Cycle

Figure 6: eDRX Mode (i.e., C-DRX = 0, PSM = 1, and T3324 = $4 \mathrm{~m}$ ) with BG96/Avnet shield under Operator1: (a) Power trace of UE's eDRX mode with an average power consumption of $0.071 \mathrm{~W}$; (b) Power trace of UE's eDRX cycle with an overall average power consumption of $0.070 \mathrm{~W}$; (c) Power trace of UE's PTW with an average power consumption of 0.078 W (d) Power trace of I-eDRX cycle with an average power consumption of $0.081 \mathrm{~W}$.

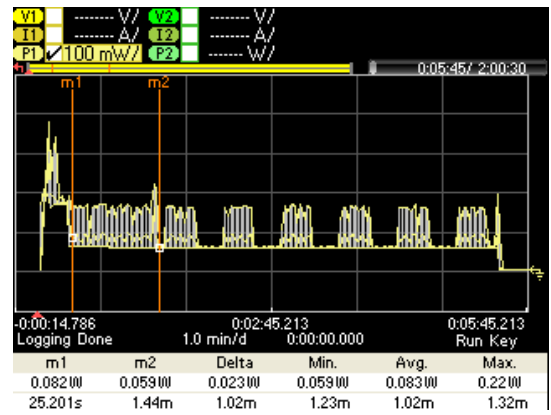

(a) C-DRX Mode

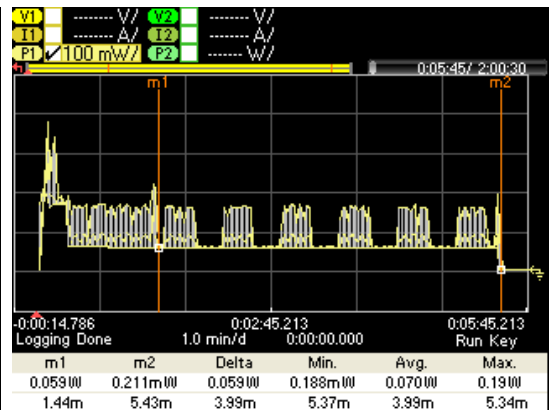

(b) eDRX mode

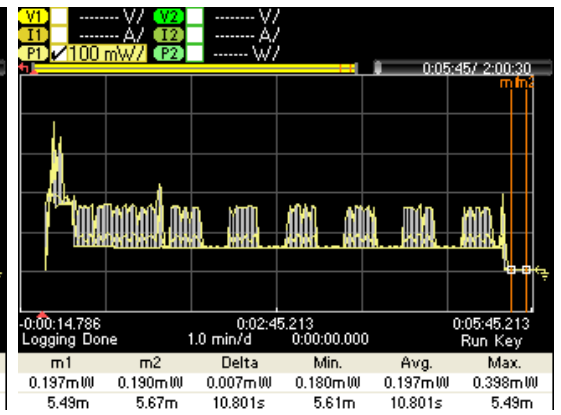

(c) PSM

Figure 7: Power cycle with BG96/Avnet shield under Operator1 network: (a) C-DRX mode runs for 1.0 min (UE configured), (b) eDRX mode runs for 4 min (UE-configured), and (c) PSM runs for $60 \mathrm{~min}$ (UE-configured), not shown in full for readability.

traces from these experiments are shown in Figures 11a and 11b. It was observed that the radio consumed $0.00052 \mathrm{Wh}$ at $\mathrm{CEL}=0$ and $0.00080 \mathrm{Wh}$ at $\mathrm{CEL}=1$ for the transmission of the same 71 Bytes of data through the HTTPS protocol, i.e. an increase of $53.8 \%$ in the energy consumption when the CEL changed i.e, the radio transmits for longer time because of the lower signal strength. In comparison to the UDP transmission protocol, this was an increase of $372 \%$ and $400 \%$ at CEL $=0$ and CEL $=1$, respectively, because of transmitting the extra 61 bytes of data overhead.

Tables IV and V summarize the power consumption of various states of the Avnet Silica BG96 shield under
Operator1 and Operator2 test networks, respectively.

5) Verifying our results for Operatorl and Operator 2 networks with Quectel BG96 EVB Kit: All the above experiments were repeated for both the operators on the same location and under similar conditions using the Quectel BG96 EVB kit [30]. Since similar power graphs for C-DRX, eDRX, and PSM modes of the BG96 radio were obtained from the PA, these graphs are not included in the paper for conciseness. Nevertheless, the results obtained for all these tests are summarized in Tables VI and VII, respectively.

Finally, a side-by-side comparison of the current and power consumption of the two boards i.e., Avnet BG96 shield and 


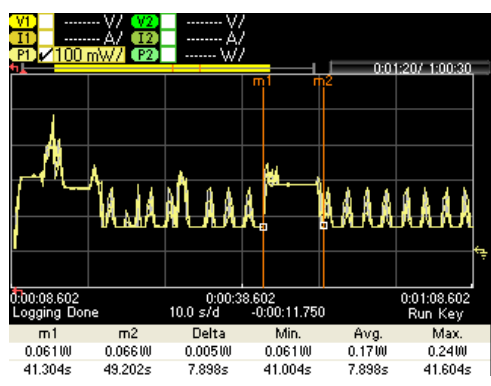

(a) Data Transmission at CEL $=0$

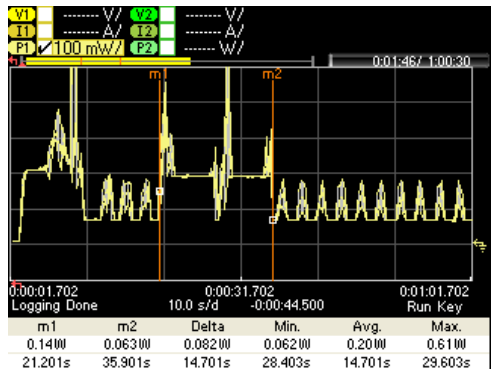

(b) Data Transmission at $\mathrm{CEL}=1$

Figure 8: Transmitting 10 bytes of data using UDP protocol on Operator1 network. (a) Data Transmission at CEL $=0$ consumes $0.17 \mathrm{~W}$ for $7.898 \mathrm{~s}(0.000372 \mathrm{Wh})$, (b) Data Transmission at $\mathrm{CEL}=1$ consumes $0.20 \mathrm{~W}$ for $14.701 \mathrm{~s}(0.000816 \mathrm{Wh})$, i.e. an increase of $119.35 \%$.

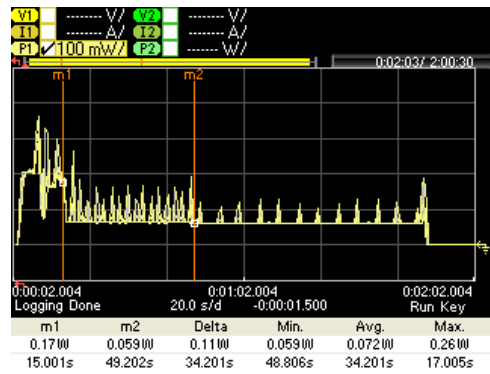

(a) C-DRX mode of $34 \mathrm{~s}$

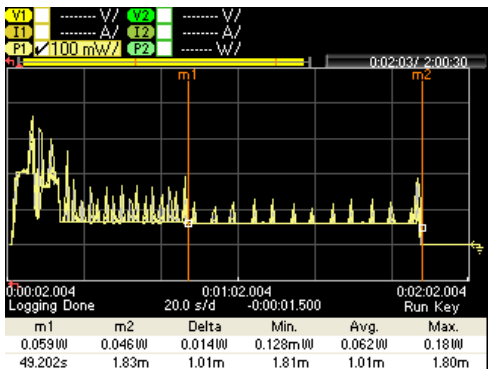

(b) eDRX mode

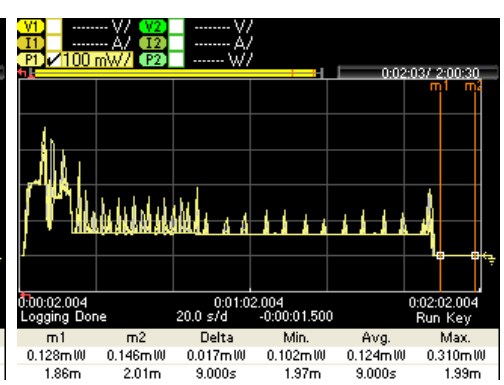

(c) PSM

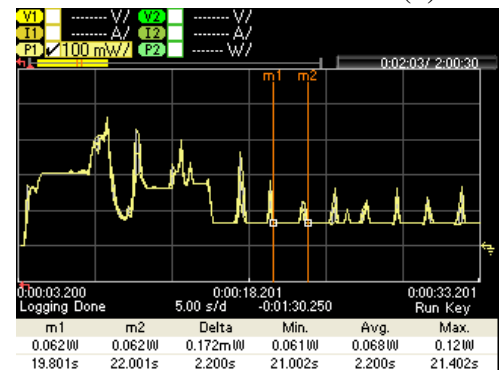

(d) C-DRX Cycle

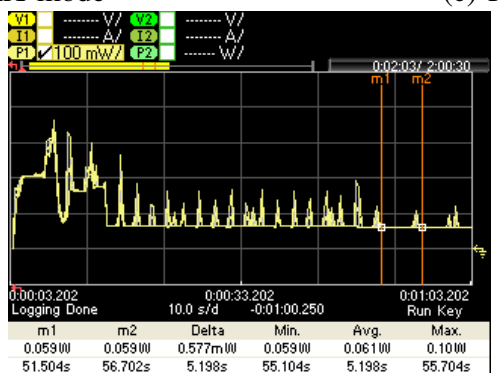

(e) I-DRX Cycle

Figure 9: Power cycle of the Avnet BG96 shield under Operator2 network: (a) C-DRX mode runs for $34.2 \mathrm{~s}$, (b) eDRX mode runs for $1.0 \mathrm{~min}$ (UE configured), and (c) PSM runs for $1.0 \mathrm{~h}$ (UE configured), not shown in full for readability. In Operator 2 network, the C-DRX Cycle is $2.1 \mathrm{~s}$ while the I-DRX Cycle is $5.12 \mathrm{~s}$.

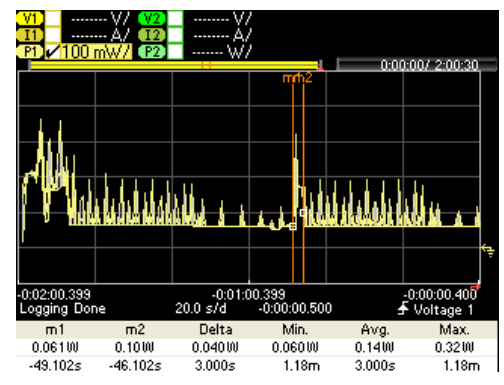

(a) Data transmission at $\mathrm{CEL}=0$

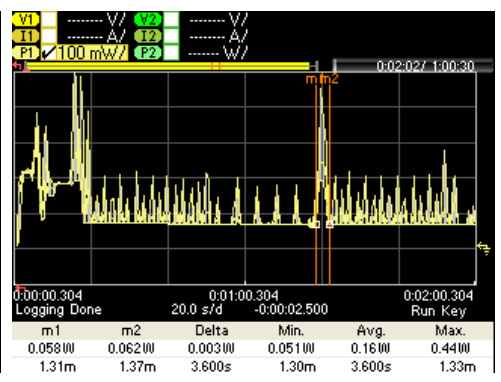

(b) Data transmission at $\mathrm{CEL}=1$

Figure 10: Transmitting 10 byte of data using UDP protocol on Operator 2 network. (a) Data Transmission at CEL $=0$ consumes $0.14 \mathrm{~W}$ for $3 \mathrm{~s}(0.00011 \mathrm{Wh})$, (b) Data Transmission at CEL=1 consumes $0.16 \mathrm{~W}$ for $3.6 \mathrm{~s}(0.00016 \mathrm{Wh})$, i.e. an increase of $45.45 \%$.

Quectel BG96 EVB kit, for both the networks i.e, Operator 1 and Operator 2, are summarized in Tables VIII and Tables IX.

\section{Summary and discussion of the measurement results}

In the remainder of this section, we summarize our main observations of the experimental results and present a discussion 


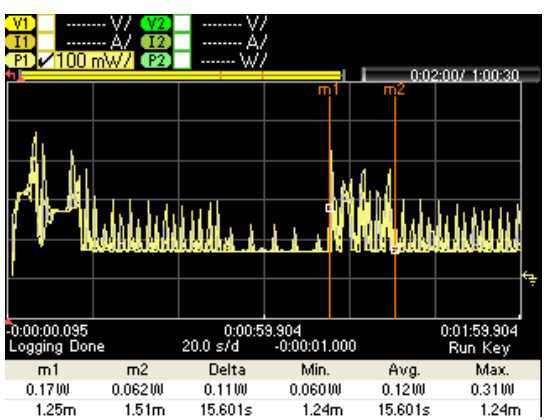

(a) Data transmission at $\mathrm{CEL}=0$

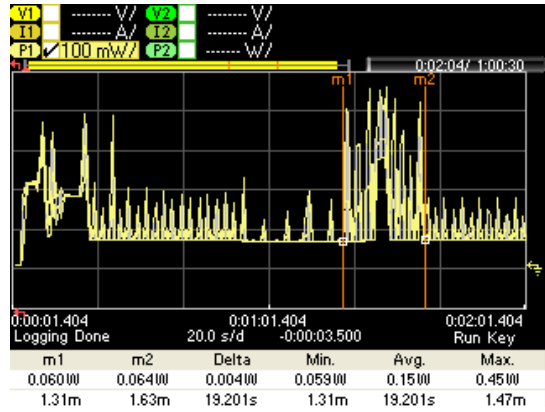

(b) Data transmission at $\mathrm{CEL}=1$

Figure 11: Transmitting 71 bytes of data to ThingSpeak server [35] using HTTPS protocol on Operator2 network. (a) Data Transmission at $\mathrm{CEL}=0$ consumes $0.12 \mathrm{~W}$ for $15.6 \mathrm{~s}(0.00052 \mathrm{Wh})$, (b) Data Transmission at $\mathrm{CEL}=1$ consumes $0.15 \mathrm{~W}$ for $19.2 \mathrm{~s}(0.0008 \mathrm{Wh})$, i.e. an increase of $53.84 \%$.

Table IV: Summary of the power consumption of various states of the Avnet Silica BG96 shield under Operator1 network

\begin{tabular}{|c|c|c|}
\hline \multicolumn{3}{|c|}{ Avnet Silica BG96 shield current and power consumption details with a constant 3.3V power supply } \\
\hline Operational Modes & Avg Current & Avg Power \\
\hline Attach/Resume Procedure $(\approx \mathbf{1 8 s})$ & $56.8 \mathrm{~mA}$ & $180 \mathrm{~mW}$ \\
\hline \multirow{4}{*}{$\begin{array}{l}\text { C-DRX Mode(Not fixed to any value) } \\
\text { C-DRX Cycle }=2.56 \mathrm{~s} \\
\text { On duration }(\mathrm{PO})=1.28 \mathrm{~s} \\
\text { Off duration }(\mathrm{SP})=1.28 \mathrm{~s} \\
\end{array}$} & $25.1 \mathrm{~mA}$ & $82 \mathrm{~mW}$ \\
\hline & $25.1 \mathrm{~mA}$ & $82 \mathrm{~mW}$ \\
\hline & $32 \mathrm{~mA}$ & $110 \mathrm{~mW}$ \\
\hline & $18.1 \mathrm{~mA}$ & $59 \mathrm{~mW}$ \\
\hline \multirow{7}{*}{$\begin{array}{l}\text { eDRX Mode (as defined by T3324 }=\mathbf{4} \mathbf{~ m}) \\
\text { eDRX Cycle }=40.96 \mathrm{~s} \\
\text { PTW }=20.48 \mathrm{~s} \\
\mathrm{I}-\mathrm{eDRX} \text { Cycle }=2.56 \mathrm{~s} \\
\quad \text { On duration }(\mathrm{PO})=1.28 \mathrm{~s} \\
\text { Off duration }(\mathrm{SP})=1.28 \mathrm{~s} \\
\text { eDRX Opportunity }=20.48 \mathrm{~s}\end{array}$} & $21.8 \mathrm{~mA}$ & $71 \mathrm{~mW}$ \\
\hline & $21.8 \mathrm{~mA}$ & $70 \mathrm{~mW}$ \\
\hline & $25.5 \mathrm{~mA}$ & $78 \mathrm{~mW}$ \\
\hline & $24.47 \mathrm{~mA}$ & $81 \mathrm{~mW}$ \\
\hline & $31 \mathrm{~mA}$ & $110 \mathrm{~mW}$ \\
\hline & $17.98 \mathrm{~mA}$ & $59 \mathrm{~mW}$ \\
\hline & $17.97 \mathrm{~mA}$ & $59 \mathrm{~mW}$ \\
\hline PSM Mode (as defined by (T3412-T3324) value) & $0.05 \mathrm{~mA}$ & $0.19 \mathrm{~mW}$ \\
\hline
\end{tabular}

Table V: Summary of the power consumption of various states of the Avnet Silica BG96 shield under Operator2 network

\begin{tabular}{|c|c|c|}
\hline \multicolumn{3}{|c|}{ Avnet Silica BG96 shield current and power consumption details with a constant $3.3 \mathrm{~V}$ power supply } \\
\hline Operational Modes & Avg Current & Avg Power \\
\hline Attach/Resume Procedure $(\approx 12 \mathrm{~s})$ & $40.1 \mathrm{~mA}$ & $190 \mathrm{~mW}$ \\
\hline \multirow{4}{*}{$\begin{array}{l}\text { C-DRX Mode (Fixed to 34 s) } \\
\text { C-DRX Cycle }=2.1 \mathrm{~s} \\
\text { On duration }(\mathrm{PO})=0.5 \mathrm{~s} \\
\text { Off duration }(\mathrm{SP})=1.6 \mathrm{~s}\end{array}$} & $21.3 \mathrm{~mA}$ & $72 \mathrm{~mW}$ \\
\hline & $21.2 \mathrm{~mA}$ & $70 \mathrm{~mW}$ \\
\hline & $28 \mathrm{~mA}$ & $98 \mathrm{~mW}$ \\
\hline & $18.6 \mathrm{~mA}$ & $62 \mathrm{~mW}$ \\
\hline \multirow{4}{*}{$\begin{array}{l}\text { eDRX Mode (as defined by T3324) } \\
\text { eDRX Cycle }=5.12 \mathrm{~s}(\text { Fixed) } \\
\text { On duration }(\mathrm{PO})=0.3 \mathrm{~s} \\
\text { Off duration }(\mathrm{SP})=4.7 \mathrm{~s}\end{array}$} & $19.2 \mathrm{~mA}$ & $63 \mathrm{~mW}$ \\
\hline & $18.8 \mathrm{~mA}$ & $62 \mathrm{~mW}$ \\
\hline & $26.2 \mathrm{~mA}$ & $87 \mathrm{~mW}$ \\
\hline & $18.2 \mathrm{~mA}$ & $60 \mathrm{~mW}$ \\
\hline PSM Mode (as defined by (T3412-T3324) value) & $0.03 \mathrm{~mA}$ & $0.12 \mathrm{~mW}$ \\
\hline
\end{tabular}

Table VI: Summary of the power consumption of various states of QUECTEL BG96 EVB Kit under Operator1 network

\begin{tabular}{|c|c|c|}
\hline \multicolumn{3}{|c|}{ QUECTEL BG96 Kit current and power consumption details with a constant 3.8V power supply } \\
\hline Operational Modes & Avg Current & Avg Power \\
\hline Attach/Resume Procedure $(\approx 18 \mathrm{~s})$ & $51.8 \mathrm{~mA}$ & $200 \mathrm{~mW}$ \\
\hline \multirow{4}{*}{$\begin{array}{l}\text { C-DRX Mode(Not fixed by the operator) } \\
\text { C-DRX Cycle }=2.56 \mathrm{~s} \\
\text { On duration }(\mathrm{PO})=1.28 \mathrm{~s} \\
\text { Off duration }(\mathrm{SP})=1.28 \mathrm{~s}\end{array}$} & $26.1 \mathrm{~mA}$ & $100 \mathrm{~mW}$ \\
\hline & $25.6 \mathrm{~mA}$ & $97 \mathrm{~mW}$ \\
\hline & $30.6 \mathrm{~mA}$ & $120 \mathrm{~mW}$ \\
\hline & $20.1 \mathrm{~mA}$ & $78 \mathrm{~mW}$ \\
\hline \multirow{7}{*}{$\begin{array}{l}\text { eDRX Mode (as defined by T3324 = 4 m) } \\
\text { eDRX Cycle }=40.96 \mathrm{~s} \\
\text { PTW }=20.48 \mathrm{~s} \\
\text { I-eDRX Cycle }=2.56 \mathrm{~s} \\
\text { On duration }(\mathrm{PO})=1.28 \mathrm{~s} \\
\text { Off duration }(\mathrm{SP})=1.28 \mathrm{~s} \\
\text { eDRX Opportunity }=20.48 \mathrm{~s}\end{array}$} & $20.22 \mathrm{~mA}$ & $77 \mathrm{~mW}$ \\
\hline & $20.22 \mathrm{~mA}$ & $77 \mathrm{~mW}$ \\
\hline & $22.77 \mathrm{~mA}$ & $87 \mathrm{~mW}$ \\
\hline & $22.57 \mathrm{~mA}$ & $86 \mathrm{~mW}$ \\
\hline & $27.6 \mathrm{~mA}$ & $100 \mathrm{~mW}$ \\
\hline & $16.9 \mathrm{~mA}$ & $66 \mathrm{~mW}$ \\
\hline & $17.1 \mathrm{~mA}$ & $66 \mathrm{~mW}$ \\
\hline PSM Mode (value of (T3412-T3324)) & $0.05 \mathrm{~mA}$ & $0.20 \mathrm{~mW}$ \\
\hline
\end{tabular}

thereof.

For the results shown in Tables IV to IX, the experiments were repeated in the order of 100 times and the values were averaged accordingly. As indicated previously, Table IV 
Table VII: Summary of the power consumption of various states of QUECTEL BG96 EVB Kit under Operator2 network.

\begin{tabular}{|c|c|c|}
\hline \multicolumn{3}{|c|}{ QUECTEL BG96 kit current and power consumption details with a constant $3.8 \mathrm{~V}$ power supply } \\
\hline Operational Modes & Avg Current & Avg Power \\
\hline Attach/Resume Procedure $(\approx 12 \mathrm{~s})$ & $59.3 \mathrm{~mA}$ & $190 \mathrm{~mW}$ \\
\hline \multirow{4}{*}{$\begin{array}{l}\text { C-DRX Mode (Fixed to 34 s) } \\
\text { C-DRX Cycle }=2.1 \mathrm{~s} \\
\text { On duration }(\mathrm{PO})=0.5 \mathrm{~s} \\
\text { Off duration }(\mathrm{SP})=1.6 \mathrm{~s}\end{array}$} & $25.3 \mathrm{~mA}$ & 86 mW \\
\hline & $25.2 \mathrm{~mA}$ & $85 \mathrm{~mW}$ \\
\hline & $28 \mathrm{~mA}$ & $170 \mathrm{~mW}$ \\
\hline & $18.6 \mathrm{~mA}$ & $78 \mathrm{~mW}$ \\
\hline \multirow{4}{*}{$\begin{array}{r}\text { eDRX Mode (as defined by T3324) } \\
\text { eDRX Cycle }=5.12 \mathrm{~s}(\text { Fixed) } \\
\text { On duration }(\mathrm{PO})=0.4 \mathrm{~s} \\
\text { Off duration }(\mathrm{SP})=4.7 \mathrm{~s}\end{array}$} & $19.2 \mathrm{~mA}$ & $63 \mathrm{~mW}$ \\
\hline & $30.8 \mathrm{~mA}$ & $100 \mathrm{~mW}$ \\
\hline & $29.8 \mathrm{~mA}$ & $98 \mathrm{~mW}$ \\
\hline & $22.9 \mathrm{~mA}$ & $76 \mathrm{~mW}$ \\
\hline PSM Mode (value of (T3412-T3324)) & $0.05 \mathrm{~mA}$ & $0.19 \mathrm{~mW}$ \\
\hline
\end{tabular}

Table VIII: Side by side comparison of the average power measurements of Avnet BG96 shield and Quectel BG96 EVB Kit under Operator1 and Operator2 networks

\begin{tabular}{|c|c|c|c|c|}
\hline \multicolumn{5}{|c|}{ Power Consumption of the Avnet BG96 shield and Quectel BG96 EVB Kit } \\
\hline Avnet $\quad$ Quectel & $\operatorname{Attach}(\mathbf{m W})$ & CDRX $(\mathbf{m W})$ & eDRX $(\mathrm{mW})$ & PSM $(\mathbf{m W})$ \\
\hline Operator 1 & $180.0 \quad 200$ & 82.0 & $71.0 \quad 77.0$ & $0.19 \quad 0.20$ \\
\hline Operator 2 & $190.0 \quad 190$ & $72.0 \quad 86.0$ & $\begin{array}{ll}63.0 & 63.0\end{array}$ & 0.12 \\
\hline
\end{tabular}

Table IX: Side by side comparison of the average current measurements of Avnet BG96 shield and Quectel BG96 EVB Kit under Operator1 and Operator2 networks

\begin{tabular}{|c|c|c|c|c|}
\hline \multicolumn{5}{|c|}{ Current consumption of the Avnet BG96 shield and Quectel BG96 EVB Kit } \\
\hline Avnet $\quad$ Quectel & Attach $(\mathbf{m A})$ & CDRX (mA) & eDRX (mA) & PSM (mA) \\
\hline Operator 1 & $\begin{array}{lr}56.8 \quad 51.8 \\
\end{array}$ & $25.1 \quad 26.1$ & $\begin{array}{ll}21.8 & 20.22 \\
\end{array}$ & $0.05 \quad 0.05$ \\
\hline Operator 2 & \begin{tabular}{|lr}
40.1 & 59.3 \\
\end{tabular} & $21.3 \quad 25.3$ & $19.2 \quad 19.2$ & $\begin{array}{ll} & 0.05 \\
& \end{array}$ \\
\hline
\end{tabular}

summarizes the current and power consumption details of the Avnet shield under Operator1's network, whereas Table $\mathrm{V}$ summarizes the current and power consumption details of the Avnet board under Operator2's network. Comparing the current and power data from both of these tables, it can be noted that with Operator1's network, the BG96 radio consumes more power on average for most of its operational modes as compared to when operating under Operator2' network. It can also be noted that contrary to the other radio modes, the power and current data values for the PSM are the same with both networks ${ }^{1}$

The same observations stand true when comparing the current and power consumption data in Table VI and VII obtained for the Quectel BG96 EVB kit for both of these networks. It is clear that the BG96 radio consumes more power on average for most of its operational modes when connected to Operator1's network as compared to Operator2.

However, comparing the current and power consumption data as obtained for both of these boards, i.e. Avnet Silica and Quectel EVB kit, it is also clear that the latter consumes more for the same network parameters and under the same network conditions.

\footnotetext{
${ }^{1}$ It is also noted that the average current consumption for PSM $=0.05 \mathrm{~mA}$ which is higher than the $0.01 \mathrm{~mA}$ value indicated in the datasheet [31]. Such difference can be due to the additional components needed to implement a BG96 minimum system on the Avnet shield (e.g. power regulator, USB interface, etc.). Such difference is also in line with our observation that, in a practical system, the energy consumption of NB-IoT radio transceivers is often under-estimated.
}

To have a better overview of all the data from the abovementioned tables, we have further summarized them in Table VIII and Table IX. All in all, it can be said that from the network side Operator1 has a higher energy consumption, while from the device side, the Quectel EVB Kit consumes more than the AVNET shield.

While the current and power consumption differences between the two boards can be explained by the fact the Quectel EVB kit features more active components than the Avnet Silica board, the differences between the two networks call for a more detailed discussion, as presented in what follows.

An essential point to keep in mind is that the UE settings affect its energy consumption to a great extent, in particular in terms of active waiting, idle waiting and PSM. At the same time, these also have a notable impact on the application QoS. In parallel, the network settings also have a significant impact on the energy consumption of the UE. In more details:

i) The inactivity timer is operator specific; thus, depending on the network configuration, this can be a major energysaving factor on the UE side. Our results have shown that Operator2 provides greater flexibility in terms of control and configurability of the C-DRX (within the inactivity timer) mode as compared to Operator1. On the other hand, Operator1 does not limit the length of its active waiting period (within the inactivity timer). This explains why Operator 1 consumes more as compared to Operator 2 since 
the latter has a controlled active waiting period. Moreover, since the inactivity timer is reset after each downlink data exchange, the longer its span the larger its impact on the UE energy consumption. Similarly, if downlink data is received in fragments, the energy consumption due to the inactivity timer will add-up.

ii) The activity timer is UE configurable, but its underneath eDRX cycles with its PTW and its underneath I-DRX cycles are network specific; thus, their settings affect the overall energy-consumption of the UE. Operator2 also provides greater flexibility in terms of control and configurability of its eDRX settings as compared to Operator1; since the former supports more robust settings for these parameters, it is thus more energy-friendly from the UE perspective. However, the effects of such parameters on the QoS of application are still unknown and beyond the scope of this paper. Though Operator 1 provides more flexibility in these settings, the overall energy consumption of the radio is higher.

iii) The power consumption of the PSM of the radio is nearly identical with both operators. This can be explained by the fact that when in the PSM mode, most parts of the radio module are turned off and no operator specific parameter affect the current drawn by the chip. However, a general comment is that while the longer the radio stays in PSM the larger its energy savings, this translates in increased latency cost and thus possibly reduced QoS for the application. This important trade-off in NB-IoT is not yet fully explored in the literature.

iv) Our experiments have also shown that the transmission power varies with the signal strength of the radio and thus affects the UE energy consumption. The transmit power can be ramped-up to a maximum of $23 \mathrm{dBm}$, whether when connecting to the BS or while transmitting data. For example, in Figure 11a it can be seen that the power for data transmission is $0.12 \mathrm{~W}$ (i.e. $20.79 \mathrm{dBm}$ ) and $0.15 \mathrm{~W}$ (i.e. $21.76 \mathrm{dBm}$ ) in Figure 11b. Since the UE has no provision to control its transmit power, the energy consumption from the UE transmit power point of view is not an exclusive UE feature.

v) The data transmission protocol varies in terms of their control overheads, data payloads, coverage level, and security/guarantees. These various aspects yield different energy consumption as seen in our experimental results when transmitting data with the UDP and HTTPs protocols in two different coverage classes. For example, Figures 8, 10 show that transiting from coverage level $\mathrm{CEL}=0$ to $\mathrm{CEL}=1$ with UDP leads to energy consumption increases between $45 \%$ and $119 \%$, i.e. up to more than a factor 2. Figure 11a and Figure $11 \mathrm{~b}$ show that the same transition with HTTPs leads to an increase of $53.84 \%$, i.e. slightly more than a factor 1.5 . Also, as mentioned earlier, the increase between UDS and HTTPs ranges from $372 \%$ and $400 \%$.

Moreover, from the results obtained through these ex- periments, it is clear that almost all of the 3GPP defined UE states are attainable on both MNO's test networks, and thus by extension on commercial networks; this is in stark contrast to what has been reported in most of the existing literature so far. The results also indicates that all the power saving features of the NB-IoT technology are included in the considered CoTS NB-IoT radio chips and could be utilized as per the application requirements. However, as the hardware and software developments of NB-IoT are ongoing, special care must be taken to choose the right firmware for the right hardware that is being used for the specific application. Our results also show that all the timers are flexible and can be set as per the 3GPP standards provided the network operators allow any such provisions from the network side and this should be kept in mind by application developers to obtain network access.

\section{Evaluation of our Proposed Model}

Section IV has presented individual empirical measurement results for various timings for the different states of the NBIoT radio module for different power saving schemes. Next, in this section we A) evaluate the error of our proposed model by calculating the difference between the energy consumption obtained from the real life deployment versus that predicted by the model and B) we summarize the sensitivity analysis conducted to evaluate which parameters have the largest impact on the total energy consumption in our proposed model.

\section{A. Model evaluation tests}

We have conducted three sets of experiments of which the base cycle lasts from $12.3 \mathrm{~min}$ to $1.2 \mathrm{~h}$ and is repeated from 2 to 10 times during the observation window. Doing so forces the NB-IoT radio in various operational conditions and allows characterizing the average differences between the energy consumption predicted by the model and the real-life values. The three sets of experiments use the Avnet BG96 shield operating on Operator1 or Operator 2 network, as described in what follows.

The first evaluation test was executed with an Avnet BG96 shield board operating on Operator1 network. The test consisted of a base power cycle of $30 \mathrm{~min}$ as captured between $\mathrm{m} 1$ and $\mathrm{m} 2$ (29.55 $\mathrm{min}$ shown) in Figure 12a and repeated twice in an observation window of $1 \mathrm{~h}$ (59.53 min shown) between $\mathrm{m} 1$ and $\mathrm{m} 2$, as shown in Figure 12b. As can be seen in Figure 12a, the base power cycle includes an attach procedure of $18 \mathrm{~s}$, and C-DRX, e-DRX and PSM states of a bit less than $10 \mathrm{~min}$ each where the average power consumption for the base power cycle is $0.052 \mathrm{~W}$. And as can be seen in Figure 12b, it is repeated twice over a period of 60 min captured between $\mathrm{m} 1$ and $\mathrm{m} 2$ (59.53 min shown) where the average power consumption is found to be $0.052 \mathrm{~W}$. The energy consumed per each power cycle as per Equation (19) is $0.022 \mathrm{Wh}$, whereas that measured with the PA is $0.026 \mathrm{Wh}$. The energy consumed for the entire observation window as per Equation (19) is $0.044 \mathrm{Wh}$, whereas that measured with the PA 
is $0.052 \mathrm{Wh}$, i.e. an error of $15.38 \%$, as indicated in Table XII.

The second evaluation test was also conducted with an Avnet BG96 shield, but this time operating on Operator2 network. The test consisted of the base power cycle shown in Figure $13 \mathrm{a}(\mathrm{m} 1$ and $\mathrm{m} 2$ in this figure are used to record the repeated C-DRX cycle of the radio after a data transmission $(\mathrm{Tx})$ ); this power cycle is repeated 3 times as shown in Figure 13b. The base power cycle lasts $12.3 \mathrm{~min}$ and includes an Attach procedure of $12.1 \mathrm{~s}$, C-DRX mode of $20 \mathrm{~s}$, Tx through UDP protocol of $3 \mathrm{~s}$, repeated C-DRX of $32 \mathrm{~s}$, eDRX of $34 \mathrm{~s}$, and PSM of a bit more than $10 \mathrm{~m}$. The base power cycle consumes on average $0.011 \mathrm{~W}$ during the $12.3 \mathrm{~min}$ duration, i.e. an average energy consumption of $0.0022 \mathrm{Wh}$. As indicated in Table XII, the energy consumed per power cycle as per Equation (19) is 0.0024 Wh, i.e. an error of $9.09 \%$.

Like the second one, the third evaluation test was conducted with the Avnet BG96 shield operating under Operator2 network, but this time for a longer duration. The base cycle lasts $1.2 \mathrm{~h}$ including an Attach procedure of 12 s, CDRX of $32 \mathrm{~s}$, e-DRX of $10 \mathrm{~min}$ and PSM of $64 \mathrm{~min}$, as shown in Figure 14a. This power cycle of $1.2 \mathrm{~h}$ has an average power consumption of $0.010 \mathrm{~W}$. It is then repeated 10 times in an observation window of $11.8 \mathrm{~h}$, as shown in Figure 14b (note that some of the PSM durations are shorter than others). In this case, the energy consumed per power cycle measured with the PA is $0.01200 \mathrm{Wh}$, whereas as per Equation (19) it is found to be $0.01204 \mathrm{Wh}$, i.e. an error of $0.33 \%$ only, as indicated in Table XII.

The error of the proposed model ranges from as low as $0.33 \%$ for longer durations (e.g. when the radio has to activate after several hours or more), and reaches up to approximately $15.38 \%$ for shorter durations (e.g. when the radio has to activate after several minutes to hours). Since the majority of NBIoT applications are intended for longer duration scenarios, the error will lie on the smaller end; as also indicated by the sensitivity analysis of the model.

Regarding system noise and environmental noise, we have used CoTS NB-IoT devices in order to create a realistic energy consumption model that reflects the practical performance of such devices, including possible inherent system noises. Furthermore, precautions (grounding of measurement equipment, etc.) were taken when carrying out our experiments, and no environmental noises were observed during our measurement campaign. Regarding interference, although there could be an impact due to inter-cell and intra-cell interferences, we have not experienced such interferences due to the limited number of devices connected to our test networks. (The issue of intercell interference has been investigated in our previous work [36] [37], i.e. 10-to-15 percent throughput can be improved while exploiting inference minimization schemes).

\section{B. Summary of the sensitivity analysis of the model}

The sensitivity analysis (SA) was carried out on both the power consumption parameters and timings parameters of the proposed model.

The SA of the power consumption parameters i.e., $\mathrm{P}_{\text {ATTACH, }}$ $\mathrm{P}_{\mathrm{TX}}, \mathrm{P}_{\mathrm{RX}}, \mathrm{P}_{\mathrm{CDRX}}, \mathrm{P}_{\mathrm{eDRX}}, \mathrm{P}_{\mathrm{PSM}}$, and $\mathrm{P}_{\mathrm{TAU}}$ of our proposed model indicates that they are technology-dependent and may vary for various chipsets. For example, typical values for the power consumption parameters of the BC95 chipset (an advanced IoT chipset from Quectel) are slightly lower than those for the BG96 chipset from the same vendor. Similarly, the values for these power parameters may also differ slightly from vendor to vendor. That is why these power parameters affect the overall energy consumption of the radio but only to a smaller extent. And since we have used BG96 chipset based modules for all our experiments in this work, we thus decided to use the values for the power consumption parameters of BG96 chipset; with a possible impact on the overall energy consumption of the radio in a descending order as shown in table X.

Table X: Sensitivity Analysis of the power consumption parameters of BG96 radio

\begin{tabular}{lllllll}
\hline \multicolumn{7}{c}{ BG96 Power Parameters } \\
\hline $\mathrm{P}_{\mathrm{TAU}}$ & $\mathrm{P}_{\text {ATTACH }}$ & $\mathrm{P}_{\mathrm{TX}}$ & $\mathrm{P}_{\mathrm{RX}}$ & $\mathrm{P}_{\mathrm{CDRX}}$ & $\mathrm{P}_{\mathrm{eDRX}}$ & $\mathrm{P}_{\mathrm{PSM}}$ \\
$0.18 \mathrm{~W}$ & $0.18 \mathrm{~W}$ & $0.17 \mathrm{~W}$ & $0.16 \mathrm{~W}$ & $0.083 \mathrm{~W}$ & $0.070 \mathrm{~W}$ & $0.0002 \mathrm{~W}$ \\
\hline
\end{tabular}

On the other hand, the timing parameters of the proposed model i.e., $\mathrm{T}_{\text {ATTACH }}, \mathrm{T}_{\mathrm{TX}}, \mathrm{T}_{\mathrm{RX}}, \mathrm{T}_{\mathrm{CDRX}}, \mathrm{T}_{\mathrm{eDRX}}, \mathrm{T}_{\mathrm{PSM}}$, and $\mathrm{T}_{\mathrm{TAU}}$ have their minimum and maximum values (as standardized by $3 \mathrm{GPP}$ ) as given in the table XI.

Table XI: Sensitivity Analysis of the timings parameters of NB-IoT radio

\begin{tabular}{|c|c|c|c|}
\hline \multicolumn{4}{|c|}{ Minimum and Maximum values for the Timing Parameters } \\
\hline $\mathrm{T}_{\mathrm{TAU}} / T_{\mathrm{ATTACH}}$ & $\mathrm{T}_{\mathrm{TX} /} T_{\mathrm{RX}}$ & $\mathrm{T}_{\mathrm{CDRX}}$ & $\mathrm{T}_{\mathrm{eDRX}} \mathrm{T}$ \\
\hline $18.6 \mathrm{~s}-\#$ of attempts & 0s-\# of transmissions & $10 s-60 s$ & $0 \mathrm{~s}-186 \mathrm{~m} 0 \mathrm{~s}-413 \mathrm{~d}$ \\
\hline
\end{tabular}

The sensitivity analysis of these timing parameters indicate that they have an impact on the total energy consumption of the radio, in a descending order, as explained below:

- First, $\mathrm{T}_{\text {ATTACH }}$ has the most impact on the overall energy consumption of the radio, especially when the radio wakes-up frequently (e.g. in patterns of a few minutes). However, in less frequent scenarios (e.g. once per day or weeks); its impact is negligible. Furthermore, the TAU procedure has almost the same effect as that of the ATTACH procedure (especially from the practical perspective).

- Secondly, the active waiting period i.e., $\mathrm{T}_{\mathrm{CDRX}}$ and the idle waiting period i.e., $\mathrm{T}_{\mathrm{eDRX}}$ have almost an equal impact on the total energy consumption of the radio. However, since the Inactivity timer is limited by the operator (usually set to lower than $60 \mathrm{~s}$ ), its impact on the total energy consumption is lower as compared to $\mathrm{T}_{\mathrm{CDRX}}$ of relatively longer durations.

- Similarly, the impact of the payload size both in $\left(\mathrm{T}_{\mathrm{TX}}\right.$, $\mathrm{T}_{\mathrm{RX}}$ ) is not significant on the total energy consumption of the radio while the radio is operating in good coverage. However, as the coverage worsens, its impact adds up as a function of the number of repetitions that an NB-IoT radio has to perform in that coverage level. 
- Finally, the effect of PSM and its benefits in terms of the total energy consumption of the radio becomes substantial only when enabled for longer durations.

\section{Vi. Conclusion and Future Directions}

NB-IoT is an emerging technology which is expected to dominate the IoT landscape in terms of wireless communication technology for massive machine type communication. Understanding the energy budget of NB-IoT is important; however, this is weakly addressed in the state of the art. The motivation of this work was thus to provide a modelling methodology for profiling the baseline energy consumption of an NB-IoT radio transceiver based on the RRC protocol standardized by $3 \mathrm{GPP}$. The proposed energy consumption model provides a detailed and realistic NB-IoT radio transceiver energy consumption model; the detailed analysis of the RRC protocol and empirical measurements illustrates the fine-grain energy consumption of the RRC protocol for two development boards operating on two MNOs test networks. Finally, the reallife empirical evaluation results show that the error of the proposed model ranges between $0.33 \%$ and $15.38 \%$. The proposed model and its evaluation ensures that it is viable to be used as a reference benchmark for NB-IoT radio communication. In future, we will explore energy consumption optimization strategies depending on the lifetime requirement of a given application; the proposed baseline energy consumption model will be used to evaluate the impact of such optimization strategies.

\section{REFERENCES}

[1] A. Rico-Alvarino, M. Vajapeyam, H. Xu, X. Wang, Y. Blankenship, J. Bergman, T. Tirronen, and E. Yavuz, "An overview of 3gpp enhancements on machine to machine communications," IEEE Communications Magazine, vol. 54, no. 6, pp. 14-21, 2016.

[2] Y.-P. E. Wang, X. Lin, A. Adhikary, A. Grovlen, Y. Sui, Y. Blankenship, J. Bergman, and H. S. Razaghi, "A primer on 3gpp narrowband internet of things," IEEE Communications Magazine, vol. 55, no. 3, pp. 117-123, 2017.

[3] P. Mekikis, K. Ramantas, A. Antonopoulos, E. Kartsakli, L. SanabriaRusso, J. Serra, D. Pubill, and C. Verikoukis, "Nfv-enabled experimental platform for $5 \mathrm{~g}$ tactile internet support in industrial environments," IEEE Transactions on Industrial Informatics, vol. 16, no. 3, pp. 1895-1903, 2020.

[4] J. Serra, L. Sanabria-Russo, D. Pubill, and C. Verikoukis, "Scalable and flexible iot data analytics: when machine learning meets sdn and virtualization," in 2018 IEEE 23rd International Workshop on Computer Aided Modeling and Design of Communication Links and Networks (CAMAD), 2018, pp. 1-6.

[5] E. U. T. R. Access, "Medium access control (mac) protocol specification (release 8)", 3gpp ts 36.321," V8. 0.0,(Dec. 2007), 2008.

[6] G. T. 36.331, "Evolved universal terrestrial radio access (e-utra); radio resource control (rrc); protocol specification (release 10)," 2012.

[7] E. U. T. R. Access, "Radio resource control (rrc), 3gpp ts 36.331," 2012.

[8] C.-W. Chang and J.-C. Chen, "Adjustable extended discontinuous reception cycle for idle-state users in lte-a," IEEE Communications Letters, vol. 20, no. 11, pp. 2288-2291, 2016.

[9] A. K. Sultania, C. Delgado, and J. Famaey, "Implementation of nb-iot power saving schemes in ns-3," in Proceedings of the Workshop on NextGeneration Wireless with ns-3, 2019, pp. 5-8.

[10] A. K. Sultania, P. Zand, C. Blondia, and J. Famaey, "Energy modeling and evaluation of nb-iot with psm and edrx," in IEEE Globecom Workshops (GC Wkshps). IEEE, 2018, pp. 1-7.

[11] G. Tsoukaneri, F. Garcia, and M. K. Marina, "Narrowband iot device energy consumption characterization and optimizations." in EWSN, 2020, pp. $1-12$.
[12] C. Y. Yeoh, A. bin Man, Q. M. Ashraf, and A. K. Samingan, "Experimental assessment of battery lifetime for commercial off-the-shelf nb-iot module," in 2018 20th International Conference on Advanced Communication Technology (ICACT). IEEE, 2018, pp. 223-228.

[13] P. Andres-Maldonado, P. Ameigeiras, J. Prados-Garzon, J. NavarroOrtiz, and J. M. Lopez-Soler, "Narrowband iot data transmission procedures for massive machine-type communications," IEEE Network, vol. 31, no. 6 , pp. 8-15, 2017.

[14] P. Andres-Maldonado, M. Lauridsen, P. Ameigeiras, and J. M. LopezSoler, "Analytical modeling and experimental validation of nb-iot device energy consumption," IEEE Internet of Things Journal, vol. 6, no. 3, pp. 5691-5701, 2019.

[15] P. Andres-Maldonado, P. Ameigeiras, J. Prados-Garzon, J. J. RamosMunoz, and J. M. Lopez-Soler, "Optimized lte data transmission procedures for iot: Device side energy consumption analysis," in 2017 IEEE International Conference on Communications Workshops (ICC Workshops). IEEE, 2017, pp. 540-545.

[16] M. El Soussi, P. Zand, F. Pasveer, and G. Dolmans, "Evaluating the performance of emtc and nb-iot for smart city applications," in 2018 IEEE International Conference on Communications (ICC). IEEE, 2018, pp. 1-7.

[17] R. Mozny, P. Masek, M. Stusek, K. Zeman, A. Ometov, and J. Hosek, "On the performance of narrow-band internet of things (nb-iot) for delaytolerant services," in 2019 42nd International Conference on Telecommunications and Signal Processing (TSP). IEEE, 2019, pp. 637-642.

[18] S. S. Basu, A. K. Sultania, J. Famaey, and J. Hoebeke, "Experimental performance evaluation of nb-iot," in 2019 International Conference on Wireless and Mobile Computing, Networking and Communications (WiMob). IEEE, 2019, pp. 1-6.

[19] B. Martinez, F. Adelantado, A. Bartoli, and X. Vilajosana, "Exploring the performance boundaries of nb-iot," IEEE Internet of Things Journal, vol. 6 , no. 3, pp. 5702-5712, 2019 .

[20] S. Duhovnikov, A. Baltaci, D. Gera, and D. A. Schupke, "Power consumption analysis of nb-iot technology for low-power aircraft applications," in 2019 IEEE 5th World Forum on Internet of Things (WF-IoT). IEEE, 2019, pp. 719-723.

[21] M. Lauridsen, R. Krigslund, M. Rohr, and G. Madueno, "An empirical nb-iot power consumption model for battery lifetime estimation," in 2018 IEEE 87th Vehicular Technology Conference (VTC Spring). IEEE, 2018, pp. $1-5$.

22] P. Jörke, R. Falkenberg, and C. Wietfeld, "Power consumption analysis of nb-iot and emtc in challenging smart city environments," in 2018 IEEE Globecom Workshops (GC Wkshps). IEEE, 2018, pp. 1-6.

[23] K. Mikhaylov, M. Stusek, P. Masek, V. Petrov, J. Petajajarvi, S. Andreev, J. Pokorny, J. Hosek, A. Pouttu, and Y. Koucheryavy, "Multi-rat lpwan in smart cities: Trial of lorawan and nb-iot integration," in 2018 IEEE International Conference on Communications (ICC). IEEE, 2018, pp. $1-6$.

[24] M. Lukic, S. Sobot, I. Mezei, D. Danilovic, and D. Vukobratovic, "Indepth real-world evaluation of nb-iot module energy consumption," arXiv preprint arXiv:2005.13648, 2020.

[25] G. T. 23.401, "3GPP TS 23.401, GPRS Enhancements for Evolved Universal Terrestrial Radio Access Network (E-UTRAN) Access," 2013.

[26] — " "3GPP, "TS 23.401 GPRS enhancements for Evolved Universal Terrestrial Radio Access Network access," Rel 14 v14.1.0, 2016," 2016.

[27] — , "3GPP, "TS 23.401 GPRS enhancements for Evolved Universal Terrestrial Radio Access Network access," Rel 16 V16.6.0 (2020-03)," 2020.

[28] T. ETSI, “123 401 v14. 3.0," 2017.

[29] Avnet:Silica, "Avnet: Quality Electronic Components Services." [Online]. Available: https://www.avnet.com/wps/portal/silica/products/newproducts/npi/2018/avnet-nb-iot-shield-sensor/

[30] Quectel UMTS LTE EVB Kit, "Quectel." [Online]. Available: https://www.quectel.com/product/umtsevb.htm

[31] Quectel LPWA IoT Module, "BG96 LTE Cat M1/NB1/EGPRS Module." [Online]. Available: https://www.quectel.com/product/bg96.htm

[32] Keysight Technologies, "N6705C DC Power Analyzer." [Online]. Available: https://www.keysight.com/en/pd-2747858-pn-N6705C/dc-poweranalyzer-modular-600-w-4-slots? $\mathrm{cc}=$ EElc=eng

[33] Digilent, "pmod-usb-to-uart-interface." [Online]. Available: https://store.digilentinc.com/pmod-usbuart-usb-to-uart-interface/

[34] Avnet-Silica-team, "NBIOTBG96SHIELD-HW-schematic." [Online]. Available: https://github.com/Avnet-Silica-team/NBIoTBG96HW/blob/master/BAENBIOTBG96SHIELD ${ }_{R} S R 1157 C$ SCHEMA.pdf /

[35] ThingSpeak Server, "ThingSpeak for IoT Projects ." [Online]. Available: https://thingspeak.com/channels/1085017/ 

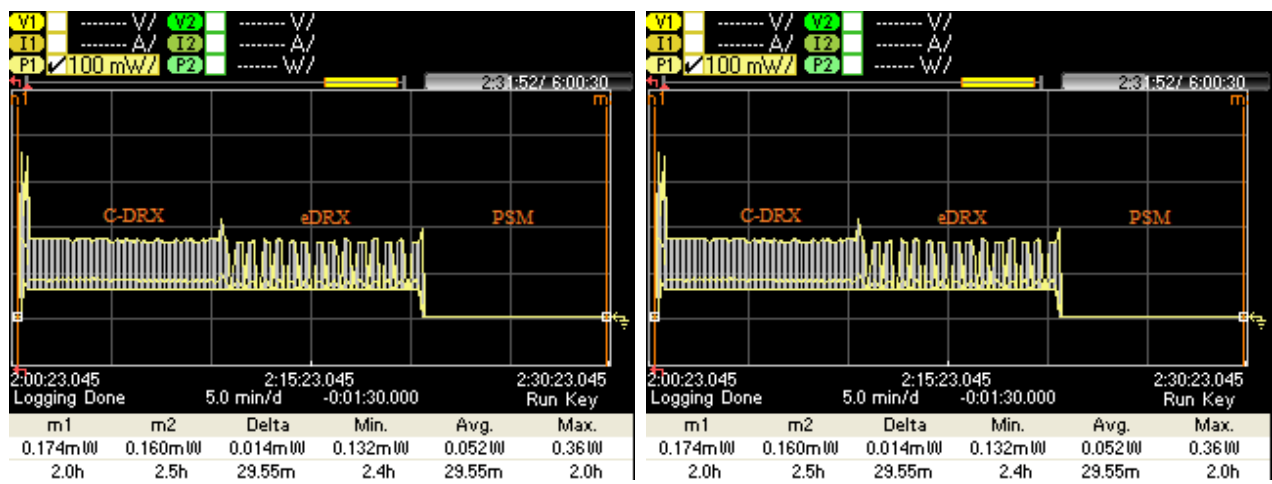

(a) A power cycle of $30 \mathrm{~min}$ ("29.55m" displayed (b) The power cycle of (a) is repeated for 2 times between $\mathrm{m} 1$ and $\mathrm{m} 2$ markers) that includes an in an observation window of $60 \mathrm{~min}$ ("59.53m" Attach procedure of $18 \mathrm{~s}, \mathrm{C}-\mathrm{DRX}$, e-DRX and displayed between $\mathrm{m} 1$ and $\mathrm{m} 2$ markers).

PSM of a bit less than 10 min each.

Figure 12: Power traces of the first evaluation test with the Avnet BG96 shield operating on Operator1 network.
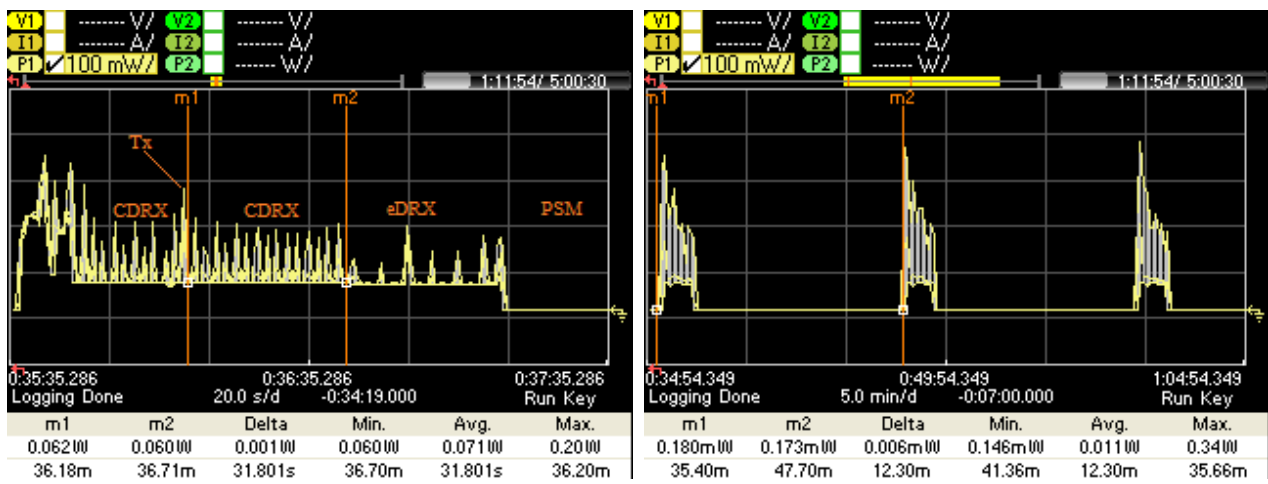

(a) A power cycle of $12.3 \mathrm{~min}$ that includes an (b) The power cycle of (a) $12.3 \mathrm{~min}$ ("12.30m" Attach procedure of $12.1 \mathrm{~s}$, C-DRX mode of $20 \mathrm{~s}$, displayed between $\mathrm{m} 1$ and $\mathrm{m} 2$ markers) is Tx ( 10 bytes data over UDP) of $3 \mathrm{~s}$, repeated C- repeated 3 times (The last PSM phase is not DRX of $32 \mathrm{~s}$ ("31.801s" displayed between $\mathrm{m} 1$ shown in full for readability)). and $\mathrm{m} 2$ markers), eDRX of $34 \mathrm{~s}$ and PSM of a bit more than $10 \mathrm{~min}$ (not shown in full for readability).

Figure 13: Power traces of the second evaluation test with Avnet BG96 shield on Operator2 network
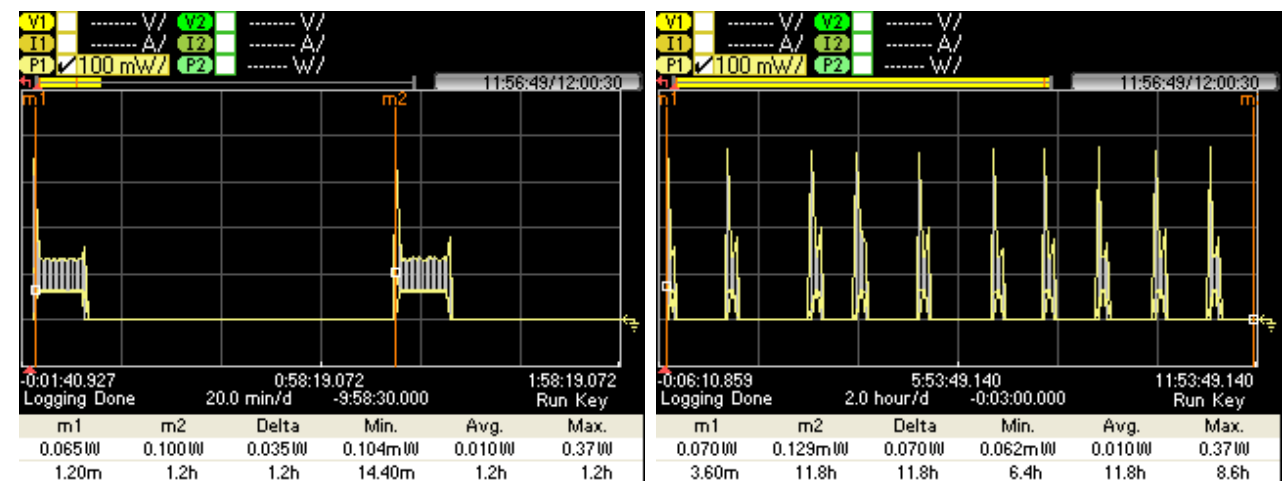

(a) A power cycle of $1.2 \mathrm{~h}$ ("1.2h" between $\mathrm{ml}$ (b) The power cycle of (a) is repeated 10 times and $\mathrm{m} 2$ markers) including an Attach procedure in an observation window of $11.8 \mathrm{~h}$ ("11.8h" of $12.1 \mathrm{~s}$, C-DRX of $32 \mathrm{~s}$, e-DRX of $10 \mathrm{~min}$ and between $\mathrm{m} 1$ and $\mathrm{m} 2$ markers). (Note that some PSM of $64 \mathrm{~min}$.

of the PSM durations are shorter than others).

Figure 14: Power traces of the third evaluation test with Avnet BG96 shield under Operator2 network. 
Table XII: NB-IoT radio energy consumption error: proposed model vs. real-life evaluation tests

\begin{tabular}{c|c|c|c}
\hline Test setup & Energy as per model (Wh) & Energy as per measurement (Wh) & Relative Error (\%) \\
\hline Avnet BG96 shield, Operator1 & 0.052 & 0.044 & 15.38 \\
\hline Avnet BG96 shield, Operator2 & 0.0024 & 0.0022 & 9.09 \\
\hline Avnet BG96 shield, Operator2 & 0.01204 & 0.01200 & 0.33 \\
\hline
\end{tabular}

[36] H. Malik, H. Pervaiz, M. M. Alam, Y. Le Moullec, A. Kuusik, and M. A. Imran, "Radio resource management scheme in nb-iot systems," IEEE Access, vol. 6, pp. 15051-15064, 2018.

[37] H. Malik, M. M. Alam, H. Pervaiz, Y. Le Moullec, A. Al-Dulaimi,
S. Parand, and L. Reggiani, "Radio resource management in nb-iot systems: Empowered by interference prediction and flexible duplexing," IEEE Network, vol. 34, no. 1, pp. 144-151, 2019. 